\title{
SINGLE CROSSING PROPERTIES AND THE EXISTENCE OF PURE STRATEGY EQUILIBRIA IN GAMES OF INCOMPLETE INFORMATION
}

\section{By SUSAN ATHEY ${ }^{1}$}

This paper analyzes a class of games of incomplete information where each agent has private information about her own type, and the types are drawn from an atomless joint probability distribution. The main result establishes existence of pure strategy Nash equilibria (PSNE) under a condition we call the single crossing condition (SCC), roughly described as follows: whenever each opponent uses a nondecreasing strategy (in the sense that higher types choose higher actions), a player's best response strategy is also nondecreasing. When the SCC holds, a PSNE exists in every finite-action game. Further, for games with continuous payoffs and a continuum of actions, there exists a sequence of PSNE to finite-action games that converges to a PSNE of the continuum-action game. These convergence and existence results also extend to some classes of games with discontinuous payoffs, such as first-price auctions, where bidders may be heterogeneous and reserve prices are permitted. Finally, the paper characterizes the SCC based on properties of utility functions and probability distributions over types. Applications include first-price, multi-unit, and all-pay auctions; pricing games with incomplete information about costs; and noisy signaling games.

KEYWORDS: Games of incomplete information, pure strategy Nash equilibrium, auctions, pricing games, signaling games, supermodular games, single crossing property.

\section{INTRODUCTION}

THIS PAPER DERIVES sufficient conditions for a class of games of incomplete information, such as first-price auction games, to have pure strategy Nash equilibria (PSNE). The class of games is 
described as follows. There is a finite number of players, each with private information about her own type. Each player's type is drawn from a convex subset of the real line. The joint distribution of types is atomless, and the types are not necessarily independent. Each player takes an action after observing her type. Players may be heterogeneous in utility functions or in the distribution of types, and the utility functions may depend directly on other players' types. Thus, the formulation includes the "mineral rights" auction (Milgrom and Weber (1982)), where bidders receive a signal about the underlying value of the object, and signals and values may be correlated across players.

The goal of this paper is to dispense with many of the assumptions required in the prior literature on existence of PSNE. Instead, we explore the consequences of a single restriction that arises naturally in a wide variety of economic applications, the single crossing condition (SCC) for games of incomplete information. A player's strategy is said to be nondecreasing if it assigns (weakly) higher actions to higher types. The SCC requires that for every player $i$, whenever each of player $i$ 's opponents uses a nondecreasing pure strategy, player $i$ 's expected payoffs satisfy Milgrom and Shannon's (1994) single crossing property. ${ }^{2}$ The SCC implies that in response to nondecreasing strategies by opponents, each player has a best response strategy that is nondecreasing.

The paper has two main objectives. First, we show that the SCC implies existence of PSNE in a range of circumstances, and second, we characterize the SCC based on properties of the primitives (utility functions and type distributions). To accomplish the first objective, we begin by showing that when a game of incomplete information satisfies the SCC, and the players are restricted to choose from a finite action set, a PSNE exists. We then show that if players' utility functions are continuous, a PSNE to the continuum-action game can be found by taking the limit 
of a sequence of PSNE of finite-action games. Thus, the existence result is constructive and suggests a computational algorithm. Finally, we extend the latter result to allocation games, such as first-price auctions, that have a particular type of discontinuity.

To characterize the SCC based on primitives, we exploit tools developed for analyzing comparative statics in stochastic problems (Athey (1998a, b)). Using these tools, we demonstrate that a wide variety of commonly studied games satisfy the SCC. In the industrial organization literature, examples include noisy signaling games (such as limit pricing with demand shocks), as well as oligopoly games with incomplete information about costs or demand. The SCC holds in first-price auction games under a wide variety of primitive conditions; it also holds in all-pay auctions and multi-unit discriminatory auctions, under somewhat more restrictive conditions. In each of these games, our results imply that a PSNE exists in nondecreasing strategies, potentially yielding empirical implications that would not follow in a mixed strategy equilibrium or an equilibrium in non-monotone strategies.

The existence theorems exploit a variety of consequences of the SCC. The SCC implies that we may search for equilibria in the space of nondecreasing strategies. Indeed, the existence theorems can be thought of as fixed point theorems tailored to the special case of nondecreasing functions. We begin by observing that in the case of finite action sets, a nondecreasing strategy is a step function, and thus can be represented by a finite vector which determines the points of strict increase of the function. This vector is referred to as the vector of "jump points." Second, we establish that when a player's expected payoffs satisfy Milgrom and Shannon's (1994) single crossing property, the set of vectors of jump points representing optimal best responses for each player is convex. When the type distribution is atomless, Kakutani's fixed point theorem can be applied to the best-response correspondence where players choose vectors of jump points. To 
treat the continuum-action case, we proceed by taking the limit of a sequence of equilibria with successively finer action sets. We make use of the fact that a sequence of uniformly bounded, nondecreasing functions has a subsequence which converges to a nondecreasing function. Finally, the results on auctions make use of the special structure of the auction game to rule out discontinuities in the limit.

The seminal work on the existence of PSNE in games of incomplete information (Radner and Rosenthal (1982), Milgrom and Weber (1985)) restricts attention to finite-action games. It proceeds by proving existence of mixed strategy equilibria in a game where players choose probability distributions over the actions, and then providing purification theorems. ${ }^{3}$ This approach is limited because mapping from a mixed strategy equilibrium, where players effectively choose probability distributions over the actions, to a pure strategy equilibrium requires independence (or, at best, conditional independence) of type distributions, and players' types must be restricted to directly affect only their own payoffs. Radner and Rosenthal (1982) provide several counter-examples of games which fail to have PSNE, in particular games where players' types are correlated.

Although results about existence of mixed strategy equilibria can be found when actions are chosen from a compact subset of the real line (Milgrom and Weber, 1985), there are several known counter-examples to existence of PSNE in this context (Khan and Sun, 1996, 1997). In the case where types are independent and payoffs are continuous, Khan and Sun (1995) have recently shown that PSNE exist when the action sets are countably infinite, but not when the action sets are uncountable. ${ }^{4}$

An alternative approach is due to Vives (1990), who shows that a sufficient condition for existence of PSNE is that the game is supermodular in the strategies, in the following sense: if 
one player's strategy increases pointwise, the best response strategies of all opponents must increase pointwise. But, the strategies themselves need not be monotone in types. Vives' condition is applicable in games where each player's payoff function is supermodular in actions, but not in the auctions and log-supermodular pricing games highlighted in this paper.

Now consider the case of games such as auctions where indivisible objects are allocated among players. The difficulty with such games is that a player's payoffs change discontinuously when she receives an object..$^{5}$ The issue of the existence of PSNE in first-price auctions with heterogeneous agents has challenged economists for many years. Recently, several authors have made substantial progress. ${ }^{6} \quad$ While the SCC is satisfied in the settings considered in the literature, ${ }^{7}$ many interesting classes of auctions are not treated by existing analyses. We provide new results about auctions where bidders may differ in utility functions or type distributions (including in the support of these distributions), the type distributions are potentially correlated (though in somewhat restrictive ways), and reserve prices may vary by bidder. We reduce the question of existence in the game, a technically challenging fixed-point problem, to the simpler question of whether the SCC holds. Verifying the SCC entails analyzing a comparative statics question for a single bidder's decision problem, and it holds by construction in many settings.

Finally, even for the auctions where existence is known, computation of equilibrium (which involves numerically solving a system of nonlinear differential equations with two boundary points) can be difficult due to pathological behavior of the system. Thus, the computational algorithm suggested by the constructive existence theorems in this paper may be of use in applications. For example, it can be used to evaluate the effects of mergers between bidders in auctions, as well as to analyze common value auctions with heterogeneous bidders, about which very little is known. 


\section{FINITE-ACTION GAMES}

Consider a game of incomplete information between $I$ players, $i=1, \ldots, I$, where each player first observes her own type $t_{i} \in T_{i} \equiv\left[t_{i}, \bar{t}_{i}\right] \subset \mathbb{R}$ and then takes an action $a_{i}$ from a compact set $\mathscr{A}_{i} \subset \mathbb{R}$.

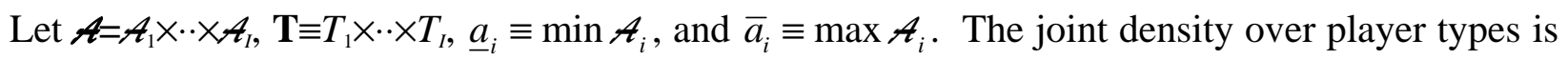
$f(\cdot)$, with the conditional density of $\mathbf{t}_{-i}$ given $t_{i}$ denoted $f\left(\mathbf{t}_{-i} \mid t_{i}\right)$. Player $i$ 's payoff function is $u_{i}: \mathcal{A} \times \mathbf{T} \rightarrow \mathbb{R}$. Given any set of strategies for the opponents, $\alpha_{j}: T_{j} \rightarrow \mathcal{A}_{j}, j \neq i$, player $i$ 's objective function is defined as follows (using the notation $\left(a_{i}, \boldsymbol{\alpha}_{-i}\left(\mathbf{t}_{-i}\right)\right) \equiv$ $\left.\left(. ., \alpha_{i-1}\left(t_{i-1}\right), a_{i}, \alpha_{i+1}\left(t_{i+1}\right), ..\right)\right)$ :

$$
U_{i}\left(a_{i}, t_{i} ; \boldsymbol{\alpha}_{-i}(\cdot)\right) \equiv \int_{t_{-i}} u_{i}\left(\left(a_{i}, \boldsymbol{\alpha}_{-i}\left(\mathbf{t}_{-i}\right)\right), \mathbf{t}\right) f\left(\mathbf{t}_{-i} \mid t_{i}\right) d \mathbf{t}_{-i}
$$

The following basic assumptions are maintained throughout the paper.

ASSUMPTION A1: The types have joint density with respect to Lebesgue measure, $f(\cdot)$, which is bounded and atomless. ${ }^{8}$ Further, $\int_{\mathbf{t}_{-i} \in S} u_{i}\left(\left(a_{i}, \alpha_{-i}\left(\mathbf{t}_{-i}\right)\right), \mathbf{t}\right) f\left(\mathbf{t}_{-i} \mid t_{i}\right) d \mathbf{t}_{-i}$ exists and is finite for all convex $S$ and all nondecreasing functions $\alpha_{j}: T_{j} \rightarrow \mathcal{A}_{j}, j \neq i$.

The following definitions are needed (the weak version will be referred to in Section 4).

DEFINITION 1: The function $h: \mathbb{R}^{2} \rightarrow \mathbb{R}$ satisfies the (Milgrom-Shannon) single crossing property of incremental returns $(S C P-I R)$ in $(x ; \theta)$ if, for all $x_{H}>x_{L}$ and all $\theta_{H}>\theta_{L}$, $h\left(x_{H}, \theta_{L}\right)-h\left(x_{L}, \theta_{L}\right) \geq(>) 0$ implies $h\left(x_{H}, \theta_{H}\right)-h\left(x_{L}, \theta_{H}\right) \geq(>) 0$, and $h$ satisfies weak SCP-IR iffor all $x_{H}>x_{L}$ and all $\theta_{H}>\theta_{L}, h\left(x_{H}, \theta_{L}\right)-h\left(x_{L}, \theta_{L}\right)>0$ implies $h\left(x_{H}, \theta_{H}\right)-h\left(x_{L}, \theta_{H}\right) \geq 0$.

The definition of SCP-IR requires that the incremental returns to $x$ cross zero at most once, from below, as a function of $\theta$. Characterizations will be given below; for the moment, note that in the special case where $h$ is differentiable, the following are sufficient for SCP-IR: 
$\frac{\partial^{2}}{\partial x \partial \theta} h(x, \theta) \geq 0$, or if $h>0, \quad \frac{\partial^{2}}{\partial x \partial \theta} \ln (h(x, \theta)) \geq 0$. The SCP-IR also admits cases such as $h(x, \theta)=u(x \theta-c(x))$, where $u$ is an increasing function. It implies that the set of optimizers is nondecreasing in the Strong Set Order, defined as follows.

DEFINITION 2: A set $A \subseteq \mathbb{R}$ is greater than a set $B \subseteq \mathbb{R}$ in the strong set order, written $A \geq_{s} B$, if, for any $a \in A$ and any $b \in B, \max (a, b) \in A$ and $\min (a, b) \in B$. If $\mathrm{K}$ is a partially ordered set, a setvalued function $A: \mathrm{K} \rightarrow 2^{\mathbb{R}}$ is nondecreasing in the strong set order if for any $\kappa_{H}>\kappa_{L}$, $A\left(\kappa_{H}\right) \geq_{S} A\left(\kappa_{L}\right)$.

LEMMA 1: (Milgrom and Shannon, 1994) Let $h: \mathbb{R}^{2} \rightarrow \mathbb{R}$. Then $h$ satisfies SCP-IR if and only if $x^{*}(\theta, B) \equiv \arg \max _{x \in B} h(x, \theta)$ is nondecreasing in $\theta$ and $B$ in the strong set order.

Under SCP-IR, there might be a $x^{\prime} \in x^{*}\left(\theta_{L}\right)$ and a $x^{\prime \prime} \in x^{*}\left(\theta_{H}\right)$ such that $x^{\prime}>x^{\prime \prime}$, so that some selection of optimizers is decreasing on a region; but if this is true, then $x^{\prime} \in x^{*}\left(\theta_{H}\right)$ and $x^{\prime \prime} \in x^{*}\left(\theta_{L}\right)$ as well. Definition 1 can be used to state the sufficient condition for existence of a PSNE in nondecreasing strategies.

DEFINITION 3: The Single Crossing Condition for games of incomplete information (SCC) is satisfied if for each $i=1, . ., I$, whenever every opponent $j \neq i$ uses a strategy $\alpha_{j}:\left[\underline{t}_{j}, \bar{t}_{j}\right] \rightarrow \mathcal{A}_{j}$ that is nondecreasing, player i's objective function, $U_{i}\left(a_{i}, t_{i} ; \alpha_{-i}(\cdot)\right)$, satisfies single crossing of incremental returns $(S C P-I R)$ in $\left(a_{i} ; t_{i}\right)$.

The SCC implies that in response to nondecreasing strategies by all opponents, each player's best response correspondence is nondecreasing in the strong set order. This implies that each player has a best response strategy that is nondecreasing; however, it allows that some best response strategies might be decreasing over an interval of types where there are multiple optimal actions. 
Our first observation is that when the action set is finite, any nondecreasing strategy is a step function, and the strategy can be described simply by naming the values of the player's type $t_{i}$ at which the player "jumps" from one action to the next higher action.

Consider the following representation. Let $\mathcal{A}_{i}=\left\{A_{0}, A_{1}, \ldots, A_{M}\right\}$ be the set of potential actions, in ascending order, where $M+1$ is the number of potential actions (and for notational simplicity, we suppose for the moment that the action sets are the same for all players). Define $T_{i}^{M} \equiv\left[t_{i}, \bar{t}_{i}\right]^{M}$, $\Sigma_{i} \equiv\left\{\mathbf{x} \in T_{i}^{M+2} \mid x_{0}=\underline{t}_{i}, x_{1} \leq x_{2} \leq \cdot \cdot \leq x_{M}, x_{M+1}=\bar{t}_{i}\right\}$, and let $\Sigma \equiv \Sigma_{1} \times \cdot \cdot \times \Sigma_{I} . \quad$ A nondecreasing strategy for player $i, \alpha_{i}: T_{i} \rightarrow \mathcal{A}_{i}$, can be represented by a vector $\mathbf{x} \in \Sigma_{i}$ according to the following algorithm (illustrated in Figure 1).

DEFINITION 4: (i) Given a nondecreasing strategy $\alpha_{i}(\cdot)$, we say that the vector $\mathbf{x} \in \Sigma_{i}$ represents $\alpha_{i}(\cdot)$ if $x_{m}=\inf \left\{t_{i} \mid \alpha_{i}\left(t_{i}\right) \geq A_{m}\right\}$ whenever there is some $n \geq m$ such that $\alpha_{i}\left(t_{i}\right)=A_{n}$ on an open interval of $T_{i}$, and $x_{m}=\bar{t}_{i}$ otherwise.

(ii) Given $\mathbf{x} \in \Sigma_{i}$, let $\{\mathbf{x}\}$ denote the set $\left\{\underline{t_{i}}, x_{1}, \ldots, x_{M}, \overline{t_{i}}\right\}$, and define $m^{*}(t, \mathbf{x}) \equiv \max \left\{m \mid x_{m}<t\right\}$. We say a nondecreasing strategy $\alpha_{i}(\cdot)$ is consistent with $\mathbf{x}$ if $\alpha_{i}\left(t_{i}\right)=A_{m^{*}\left(t_{i}, \mathbf{x}\right)}$ for all $t_{i} \in T_{i} \backslash\{\mathbf{x}\}$.

Each component of $\mathbf{x}$ is a "jump point" of the step function described by $\alpha_{i}$. Since $\mathbf{x}$ does not specify behavior for $t_{i} \in\{\mathbf{x}\}$, a given $\mathbf{x} \in \Sigma_{i}$ might correspond to more than one nondecreasing strategy. Because there are no atoms in the distributions of types, however, a player's behavior on the set $\{\mathbf{x}\}$ (which has measure zero) will not affect the best responses of other players.

Given $\mathbf{X}=\left(\mathbf{x}^{1}, . ., \mathbf{x}^{L}\right) \in \Sigma$, let $V_{1}\left(a_{1}, t_{1} ; \mathbf{X}\right)$ denote the expected payoffs to player 1 with type $t_{1}$ when player 1 chooses $a_{1} \in \mathcal{A}_{1}$ and players $2, . ., I$ use strategies consistent with $\left(\mathbf{x}^{2}, \ldots, \mathbf{x}^{I}\right)$. Then, 


$$
V_{1}\left(a_{1}, t_{1} ; \mathbf{X}\right) \equiv \sum_{m_{2}=0}^{M} . \cdot \sum_{m_{I}=0}^{M} \int_{t_{2}=x_{m_{2}}^{2}}^{x_{m_{2}+1}^{2}} \ldots \int_{t_{I}=x_{m_{I}}^{I}}^{x_{m_{I+1}}^{I}} u_{1}\left(a_{1}, A_{m_{2}}, . ., A_{m_{I}}, \mathbf{t}\right) \cdot f\left(\mathbf{t}_{-1} \mid t_{1}\right) d \mathbf{t}_{-1},
$$

and likewise for the other players.

Since (1) embeds the assumption that opponent strategies are nondecreasing, the SCC implies that $V_{i}\left(a_{i}, t_{i} ; \mathbf{X}\right)$ satisfies the SCP-IR in $\left(a_{i} ; t_{i}\right)$ for all $\mathbf{X} \in \Sigma$. Let $a_{i}^{B R}\left(t_{i} \mid \mathbf{X}\right) \equiv \arg \max _{a_{i} \in A_{i}} V_{i}\left(a_{i}, t_{i} ; \mathbf{X}\right)$; this is nonempty for all $t_{i}$ by finiteness of $\mathcal{A}_{i}$. By Lemma $1, a_{i}^{B K}(\cdot \mid \mathbf{X})$ is nondecreasing in the strong set order, which in turn implies (see Milgrom and Shannon, 1994) that there exists a selection $\gamma_{i}(\cdot)$, where $\gamma_{i}\left(t_{i}\right) \in a_{i}^{B R}\left(t_{i} \mid \mathbf{X}\right)$ for each $t_{i} \in T_{i}$, which is nondecreasing. Using Definition $4, \gamma_{i}(\cdot)$ can be represented by a $\mathbf{y} \in \Sigma_{i}$. Now define the set of all vectors that represent best response strategies:

$$
\Gamma_{i}(\mathbf{X})=\left\{\mathbf{y} \in \Sigma_{i}: \exists \alpha_{i}(\cdot) \text { which is consistent with } \mathbf{y} \text { such that } \forall t_{i} \in T_{i}, \alpha_{i}\left(t_{i}\right) \in a_{i}^{B R}\left(t_{i} \mid \mathbf{X}\right)\right\}
$$

The existence proof proceeds by showing that a fixed point exists for this correspondence. A critical property required of $\Gamma=\left(\Gamma_{1}, \ldots, \Gamma_{I}\right)$ for this purpose is convexity. Establishing this property requires some additional work, since the player might be indifferent between two actions over a set of types (and this remains true even under the additional assumptions that the player's payoff function is strictly quasi-concave and that payoffs are nowhere constant in her type, so long as the action set is finite).

In Figure $2, a_{i}^{B R}(\cdot \mid \mathbf{X})$ is nondecreasing in the strong set order. In the figure, $\mathbf{x}$ and $\mathbf{y}$ are both vectors of jump points representing optimal behavior; the arrows in the figure show convex combinations of $x_{m}$ and $y_{m}$ for $m=1, \ldots, 4$. Notice that any such convex combination also represents optimal behavior. The following Lemma shows that convexity of the best response correspondence is a general consequence of the strong set order.

LEMMA 2: $\Gamma_{i}$ is convex if $a_{i}^{B R}(\cdot \mid \mathbf{X})$ is nondecreasing in the strong set order. 
PROOF: Fix $\mathbf{X}$ and suppose that $\mathbf{w}, \mathbf{y} \in \Gamma_{i}(\mathbf{X})$. Let $\mathbf{z}=\lambda \mathbf{w}+(1-\lambda) \mathbf{y}$ for $\lambda \in(0,1)$, and observe that $\mathbf{z} \in \Sigma_{i}$. Now, for $m=0, . ., M$, we show that $A_{m}$ is an optimal action on $\left(z_{m}, z_{m+1}\right)$. If $w_{m}=w_{m+1}$ and $y_{m}=y_{m+1}$, then $z_{m}=z_{m+1}$ and there is nothing to show; so, assume that $y_{m}<y_{m+1}$.

Consider $t_{i} \in\left(z_{m}, z_{m+1}\right)$ and a $k$ such that $A_{k} \in a_{i}^{B R}\left(t_{i} \mid \mathbf{X}\right)$. Case 1: Either $w_{m}<w_{m+1}$ or $y_{m}<w_{m}=w_{m+1}<y_{m+1}$. By definition of $\mathbf{w}$ and $\mathbf{y}$, there exists a $t_{i}^{\prime}<t_{i}$ and a $t_{i}^{\prime \prime}>t_{i}$ such that $A_{m} \in a_{i}^{B R}\left(t_{i}^{\prime} \mid \mathbf{X}\right)$ and $A_{m} \in a_{i}^{B R}\left(t_{i}^{\prime \prime} \mid \mathbf{X}\right)$. If $k<m$, then $a_{i}^{B R}\left(t_{i} \mid \mathbf{X}\right) \geq_{\mathrm{S}} a_{i}^{B R}\left(t_{i}^{\prime} \mid \mathbf{X}\right)$ and $A_{m} \in a_{i}^{B R}\left(t_{i}^{\prime} \mid \mathbf{X}\right)$ imply that $A_{m} \in a_{i}^{B R}\left(t_{i} \mid \mathbf{X}\right)$. Likewise, if $k>m$, then $a_{i}^{B R}\left(t_{i}{ }^{\prime \prime} \mid \mathbf{X}\right) \geq_{\mathrm{S}} a_{i}^{B R}\left(t_{i} \mid \mathbf{X}\right)$ and $A_{m} \in a_{i}^{B R}\left(t_{i}{ }^{\prime \prime} \mid \mathbf{X}\right)$ imply that $A_{m} \in a_{i}^{B R}\left(t_{i} \mid \mathbf{X}\right)$. Case 2: $w_{m}=w_{m+1} \leq y_{m}<y_{m+1}$. Then, there exists a $t_{i}^{\prime}<t_{i}$ and an $m^{\prime \prime}>m$ such that $A_{m}^{\prime \prime} \in a_{i}^{B R}\left(t_{i}^{\prime} \mid \mathbf{X}\right)$, and a $t_{i}^{\prime \prime}>t_{i}$ such that $A_{m} \in a_{i}^{B R}\left(t_{i}^{\prime \prime} \mid \mathbf{X}\right)$. If $k<m$, then $a_{i}^{B R}\left(t_{i} \mid \mathbf{X}\right) \geq_{\mathrm{S}} a_{i}^{B R}\left(t_{i}^{\prime} \mid \mathbf{X}\right)$ and $A_{m^{\prime}} \in a_{i}^{B R}\left(t_{i}^{\prime} \mid \mathbf{X}\right)$ imply that $A_{m}{ }^{\prime} \in a_{i}^{B R}\left(t_{i} \mid \mathbf{X}\right)$. But this fact, together with $a_{i}^{B R}\left(t_{i}^{\prime \prime} \mid \mathbf{X}\right) \geq_{\mathrm{S}} a_{i}^{B R}\left(t_{i} \mid \mathbf{X}\right)$ and $A_{m} \in a_{i}^{B R}\left(t_{i}^{\prime \prime} \mid \mathbf{X}\right)$, implies that $A_{m} \in a_{i}^{B R}\left(t_{i} \mid \mathbf{X}\right)$. If $k>m, a_{i}^{B R}\left(t_{i}{ }^{\prime \prime} \mid \mathbf{X}\right) \geq_{\mathrm{S}} a_{i}^{B R}\left(t_{i} \mid \mathbf{X}\right)$ and $A_{m} \in a_{i}^{B R}\left(t_{i}{ }^{\prime \prime} \mid \mathbf{X}\right)$ implies that $A_{m} \in a_{i}^{B R}\left(t_{i} \mid \mathbf{X}\right)$. Case 3: $y_{m+1}<y_{m+1} \leq w_{m}=w_{m+1}$. Analogous to Case 2 .

Let $\beta_{i}\left(t_{i}, \mathbf{z}\right) \equiv A_{m^{*}\left(t_{i}, \mathbf{z}\right)}$. Then $\beta_{i}(\cdot, \mathbf{z})$ is a nondecreasing strategy consistent with $\mathbf{z}$ which assigns optimal actions to almost every type, implying that $\mathbf{z} \in \Gamma_{i}(\mathbf{X})$.

With convexity established, it is straightforward to prove existence of a fixed point.

LEMMA 3: Suppose that Al and the SCC hold. Then there exists a fixed point of the correspondence $\left(\Gamma_{1}, . ., \Gamma_{I}\right): \Sigma \rightarrow \Sigma$.

PROOF: Since $\Sigma$ is a compact, convex subset of $\mathbb{R}^{I(M+2)}$, we can apply Kakutani's fixed point theorem. We argued in the text that $\Gamma$ is nonempty, and Lemma 2 established convexity. We next establish that $\Gamma$ has a closed graph. From (1) and our assumption that the type distribution is atomless, it follows that $V_{i}\left(a_{i} ; \mathbf{X}, t_{i}\right)$ is continuous in the elements of $\mathbf{X}$. Consider a 
sequence $\left(\mathbf{X}^{k}, \mathbf{Y}^{k}\right)$ which converges to $(\mathbf{X}, \mathbf{Y})$, such that $\mathbf{Y}^{k} \in \Gamma\left(\mathbf{X}^{k}\right)$ for all $k$. To see that $\mathbf{Y} \in \Gamma(\mathbf{X})$, consider player $i$, and a type $t_{i} \in T_{i} \backslash\left\{\mathbf{y}^{i}\right\}$. Then there exists an $m \in\{0, . ., M\}$ such that $y_{m}^{i}<t_{i}<y_{m+1}^{i}$. Since $\mathbf{y}^{i, k}$ converges to $\mathbf{y}^{i}$, there must exist an $K$ such that, for all $k>K, y_{m}^{i, k}<t_{i}<y_{m+1}^{i, k}$, and thus $A_{m}$ is one of $t_{i}$ 's best responses to $\mathbf{X}^{k}$. Because $V_{i}\left(a_{i} t_{i} ; \mathbf{X}\right)$ is continuous in $\mathbf{X}$, if $V_{i}\left(A_{m}, t_{i} ; \mathbf{X}^{k}\right) \geq V_{i}\left(A_{m^{\prime}}, t_{i} ; \mathbf{X}^{k}\right)$ for all $k>K$ and all $m^{\prime}$, then $V_{i}\left(A_{m}, t_{i} ; \mathbf{X}\right) \geq V_{i}\left(A_{m^{\prime}}, t_{i} ; \mathbf{X}\right)$. Q.E.D.

Existence of a PSNE follows directly from this Lemma. It remains only to assign strategies to players that are consistent with a fixed point of $\Gamma$. Let $\mathbf{X}$ be such a fixed point, and let $\left(\beta_{1}(\cdot), . ., \beta_{I}(\cdot)\right)$ be a vector of nondecreasing strategies consistent with $\mathbf{X}$. Since the type distribution is atomless, a given player $i$ does not care about the behavior of her opponents at jump points, and thus $\beta_{i}(\cdot)$ is a best response to any set of strategies $\beta_{-i}(\cdot)$ consistent with $\mathbf{X}_{-i}$. This implies that $\left(\beta_{1}(\cdot), . ., \beta_{I}(\cdot)\right)$ is a PSNE of the original game. Formally:

THEOREM 1: Assume Al and the SCC hold. If $\mathscr{A}_{i}$ is finite for all $i$, this game has a PSNE where each player's equilibrium strategy, $\beta_{i}(\cdot)$, is nondecreasing.

Before proceeding, it is useful to consider the precise role of the SCC in this analysis. Can the approach be extended to non-monotone strategies? In the working paper (Athey, 1997), this question is explored more fully by considering strategies of "limited complexity." The basic idea is that a PSNE exists if we can find bounds on the "complexity" (formalized as the number of times the function changes from nondecreasing to nonincreasing or vice versa) of each player's strategy, and further, player $i$ 's best response stays within her specified bound whenever all opponents use strategies within their respective bounds. The main limitation of the extension to games with limited complexity is that much stronger assumptions may be required to guarantee convexity of the best response correspondence, which followed above (in Lemma 2) as a 
consequence of the SCC. For example, the extension in Athey (1997) requires the assumption that the best response action is unique for almost all types.

What is ruled out by the SCC, or more generally by a restriction like "limited complexity"? An example of a game with no PSNE, due to Radner and Rosenthal (1982), is an incompleteinformation version of "matching pennies." The setup is as follows: the game is zero-sum, and each player chooses actions from $\left\{A_{0}, A_{1}\right\}$. When the players choose the same action, player 2 pays $\$ 1$ to player 1 , while if the actions do not match, the players each receive zero. The types do not directly affect payoffs, and are uniformly distributed on the triangle $0 \leq t_{1} \leq t_{2} \leq 1$. Notice first that this game fails the SCC. When player 2 uses a nondecreasing strategy, player 1's best response is nondecreasing. But, when player 1 uses a nondecreasing strategy, player 2's best response is nonincreasing, since player 2 prefers not to match with player 1 . Further, this game also fails the more general "limited complexity" condition. Intuitively, player 1's best response to any pure strategy of player 2 will essentially mirror player 2's strategy, while player 2 will wish to "reverse" any pure strategy of player 1.

\section{Games with a ContinuUm of Actions and Continuous Payoffs}

\subsection{Existence of PSNE}

This section shows that the results about existence in games with a finite number of actions can be used to construct equilibria of games with a continuum of actions. The properties of the equilibrium strategies implied by the SCC play a special role in the limiting arguments used in section. While arbitrary sequences of functions need not have convergent subsequences, sequences of uniformly bounded, nondecreasing functions do have convergent subsequences. Thus, all that remains is to show that the limits of these sequences are in fact equilibria to the continuous-action game. 
The assumption of finite actions in Section 2 plays two roles: (i) it guarantees that an optimal action exists for every type, and (ii) it simplifies the description of strategies so that they can be represented with finite-dimensional vectors. In moving to the continuum-action case, we introduce the assumption that payoffs are continuous in actions. Continuity is a substitute for finiteness in (i), and further, it is used in showing that the limit of a sequence of equilibria in finite-action games is an equilibrium of the limiting game. ${ }^{9}$

THEOREM 2: Assume A1. Suppose that (i) for all $i, A_{i}=\left[a_{i}, \bar{a}_{i}\right]$, (ii) for all $i, u_{i}(\mathbf{a}, \mathbf{t})$ is continuous in a, and (iii) for any finite $\mathscr{A} \subset \mathcal{A}$, a PSNE exists in nondecreasing strategies. Then a PSNE exists in nondecreasing strategies in the game where players choose actions from $\mathcal{A}$.

PROOF: For each player $i$, consider a sequence of action sets $\left\{\mathcal{A}_{i}^{n}\right\}$, where $\mathscr{A}_{i}^{n}=\left\{\underline{a_{i}}+\frac{m}{10^{n}}\left(\bar{a}_{i}-\underline{a_{i}}\right): m=0, \ldots, 10^{n}\right\} . \quad$ Let $\mathscr{A}^{n}=\left(\mathscr{A}_{1}^{n}, \ldots, \mathcal{A}_{I}^{n}\right)$, and let $\boldsymbol{\beta}^{n}(\cdot)$ be the corresponding nondecreasing PSNE strategies. Helly's Selection Theorem (Kolmogorov and Fomin, 1970, p. 373) guarantees that a sequence of nondecreasing, uniformly bounded functions on $T_{i} \subseteq \mathbb{R}$ has a subsequence which converges to a nondecreasing function. Let $\{n\}$ denote a sequence such that $\beta^{n}(\cdot)$ converges to $\beta^{*}(\cdot)$.

Consider player $i$, and a type $t_{i} \in T_{i}$. Let $b=\beta_{i}^{*}\left(t_{i}\right)$; Helly's Selection Theorem implies $\beta_{i}^{n}\left(t_{i}\right)$ converges to $b$. Consider any $a^{\prime} \in \mathcal{A}$, and consider a sequence $\left\{a_{n}^{\prime}\right\}$ such that, for all $n, a_{n}^{\prime} \in \mathscr{A}_{i}^{n}$, and further $a_{n}^{\prime} \rightarrow a^{\prime}$. Since $\beta_{i}^{n}(\cdot)$ is an equilibrium strategy for any $n$, then for all $n$, $U_{i}\left(\beta_{i}^{n}\left(t_{i}\right), t_{i} ; \beta_{-i}^{n}(\cdot)\right) \geq U_{i}\left(a_{n}^{\prime}, t_{i} ; \beta_{-i}^{n}(\cdot)\right)$. Because payoffs are continuous and since $\beta_{-i}^{n}(\cdot)$ converges to $\boldsymbol{\beta}_{-i}^{*}(\cdot)$, it follows that for all $\mathbf{t}_{-i}, u_{i}\left(\beta_{i}^{n}\left(t_{i}\right), \boldsymbol{\beta}_{-i}^{n}\left(\mathbf{t}_{-i}\right), \mathbf{t}\right)$ converges to $u_{i}\left(b, \boldsymbol{\beta}_{-i}^{*}\left(\mathbf{t}_{-i}\right), \mathbf{t}\right)$ and $u_{i}\left(a_{n}^{\prime}, \boldsymbol{\beta}_{-i}^{n}\left(\mathbf{t}_{-i}\right), \mathbf{t}\right)$ converges to $u_{i}\left(a^{\prime}, \boldsymbol{\beta}_{-i}^{*}\left(\mathbf{t}_{-i}\right), \mathbf{t}\right)$. Thus, the expectations also converge, so that 
$U_{i}\left(b, t_{i} ; \beta_{-i}^{*}(\cdot)\right) \geq U_{i}\left(a^{\prime}, t_{i} ; \beta_{-i}^{*}(\cdot)\right)$. This guarantees that $\beta_{i}^{*}\left(t_{i}\right)$ is a best response to $\beta_{-i}^{*}(\cdot)$. Q.E.D.

COROLLARY 2.1: Assume Al and the SCC. Suppose that (i) for each $i, \mathcal{A}_{i}=\left[a_{i}, \bar{a}_{i}\right]$, and (ii) for all $i, u_{i}(\mathbf{a}, \mathbf{t})$ is continuous in $\mathbf{a}$. Then there exists a PSNE in nondecreasing strategies.

Corollary 2.1 establishes that the assumption of finite or countable actions can be dispensed with for the class of games that satisfies the SCC. This result contrasts with the general finding (see Khan and Sun, 1996, 7) that PSNE may not exist when the action sets are uncountable and the type distribution is atomless (Lebesgue). ${ }^{10}$ It can be readily verified that the counterexamples put forward by Khan and Sun $(1996,7)$ for this class of games fail the SCC.

\subsection{Characterizing the Single Crossing Condition in Applications}

This section characterizes the SCC in several classes of games of incomplete information. The results make use of the properties supermodularity and log-supermodularity. If $X$ is a lattice, the function $h: X \rightarrow \mathbb{R}$ is supermodular if, for all $\mathbf{x}, \mathbf{y} \in X$, $h(\mathbf{x} \vee \mathbf{y})+h(\mathbf{x} \wedge \mathbf{y}) \geq h(\mathbf{x})+h(\mathbf{y}) .{ }^{11} \quad$ A non-negative function $h: X \rightarrow \mathbb{R}$ is log-supermodular if, for all $\mathbf{x}, \mathbf{y} \in X, h(\mathbf{x} \vee \mathbf{y}) \cdot h(\mathbf{x} \wedge \mathbf{y}) \geq h(\mathbf{x}) \cdot h(\mathbf{y})$. Recall that when $h: \mathbb{R}^{n} \rightarrow \mathbb{R}$, and vectors are ordered in the usual way, Topkis (1978) proves that if $h$ is twice differentiable, $h$ is supermodular if and only if $\frac{\partial^{2}}{\partial x_{i} \partial x_{j}} h(\mathbf{x}) \geq 0$ for all $i \neq j$.

Five facts together can be used to establish our characterization theorems: (i) if $h: \mathbb{R}^{2} \rightarrow \mathbb{R}$ is supermodular or log-supermodular, then $h(x, t)$ satisfies SCP-IR in $(x ; t)$; (ii) sums of supermodular functions are supermodular, while products of log-supermodular functions are logsupermodular; (iii) if $h: \mathbb{R}^{n} \rightarrow \mathbb{R}$ is supermodular (resp. log-supermodular), then so is the function $h\left(\alpha_{1}\left(x_{1}\right), . ., \alpha_{n}\left(x_{n}\right)\right)$, where $\alpha_{i}(\cdot)$ is nondecreasing; (iv) a density is log-supermodular almost everywhere if and only if the random variables are affiliated (as defined in Milgrom and Weber, 
1982); (v) if $\mathbf{t}$ is affiliated, and further $h(x, \mathbf{t})$ is supermodular in $\left(x, t_{j}\right)$ for all $j$ (resp. $\log$ supermodular in $(x, \mathbf{t}))$, then $H\left(x, t_{i}\right)=\int_{\mathbf{a}}^{\mathbf{b}} h(x, \mathbf{t}) f\left(\mathbf{t}_{-i} \mid t_{i}\right) d \mathbf{t}_{-i}$ is supermodular in $\left(x, t_{i}\right)$ (resp. logsupermodular in $\left.\left(x, t_{i}, \mathbf{a}, \mathbf{b}\right)\right)$ (see Athey 1998a, b). ${ }^{12}$

\subsubsection{Supermodular and Log-Supermodular Games}

Many widely-studied games have supermodular payoffs, that is, strategic complementarities; examples include search models in the spirit of Diamond (1982), coordination games, and games with network externalities (Katz and Shapiro, 1986); Topkis (1979), Vives (1990), and Milgrom and Roberts (1990) provide a variety of additional examples. Further, two-player games with strategic substitutes can be treated as supermodular games, when one player's action is reordered. Games with strategic substitutes include Cournot quantity games as well as many games among duopolists who make investments (for example, in cost-reduction) prior to product market competition (see Athey and Schmutzler (1999) for numerous examples). Although such games are typically analyzed under the assumption of complete information, it is perhaps more realistic to consider the case where firms may have private information about their costs and benefits to acting. For example, in an oligopoly game, firms may have incomplete information about their rivals' production costs or demand elasticity; ${ }^{13}$ in an investment game, firms may have incomplete information about investment costs or results from prior research and development. The following result applies to such games.

THEOREM 3: Suppose ( $i)$ for all $i, u_{i}(\mathbf{a}, \mathbf{t})$ is supermodular in $\mathbf{a}$ and $\left(a_{i}, t_{j}\right), j=1, . ., I$, and (ii) the types are affiliated. Then the game satisfies the SCC. Thus, under Al, if either $A$ is finite, or else for all $i, \mathcal{A}_{i}=\left[\underline{a_{i}}, \bar{a}_{i}\right]$ and $u_{i}(\mathbf{a}, \mathbf{t})$ is continuous in $\mathbf{a}$, there exists a PSNE in nondecreasing strategies.

Theorem 3 follows from facts (i)-(v) above together with Corollary 2.1. It applies to games 
with additively separable payoffs (for example, when an investment has an additively separable cost, as when $u_{i}(\mathbf{a}, \mathbf{t})=h_{i}(\mathbf{a})-c_{i}\left(a_{i}, t_{i}\right)$ or $\left.u_{i}(\mathbf{a}, \mathbf{t})=h_{i}\left(\mathbf{a}, t_{i}\right)-c_{i}\left(a_{i}\right)\right)$, since supermodularity is preserved by sums. It should be noted that the games studied in Theorem 3 also satisfy Vives' (1990) sufficient condition for existence of PSNE; but, Theorem 3 guarantees the existence of a PSNE in nondecreasing strategies. This is not immediate, because even when existence is known and the SCC is satisfied, and even when all opponents use nondecreasing strategies, a player may have a best response strategy that is decreasing over an interval. In applications, additional empirical implications typically follow from monotonicity. For example, in an oligopoly context, a firm should receive greater market share when it has lower marginal cost.

Now consider the class of games with log-supermodular payoffs. This class does not satisfy Vives' (1990) conditions. An example is a pricing game among firms with differentiated products (we consider the case of homogeneous products in Section 4.2). For firm $i$, let $t_{i}$ denote the marginal cost of firm $i$, let $a_{i}$ denote the price, let $D^{i}(\mathbf{a})$ denote the demand to firm $i$, and suppose payoffs are given by $\left(a_{i}-t_{i}\right) \cdot D^{i}(\mathbf{a})$. Notice first that $\left(a_{i}-t_{i}\right)$ is log-supermodular. Further, for many commonly studied examples, $D^{i}(\mathbf{a})$ is log-supermodular; the interpretation is that a higher price by opponents makes demand less elastic. ${ }^{14}$ Then, payoffs are log-supermodular. This model can be used to analyze a variety of questions in industrial organization, such as how the presence of private information affects the relative profitability of a firm that is on average less efficient. The following theorem establishes existence of a PSNE for this class of games. THEOREM 4: Suppose (i) for all $i, u_{i}(\mathbf{a}, \mathbf{t})$ is nonnegative and log-supermodular in (a,t), and (ii) the types are affiliated. Then the game satisfies the SCC. Thus, under Al, if either A is finite, or else for all $i, \mathcal{A}_{i}=\left[a_{i}, \bar{a}_{i}\right]$ and $u_{i}(\mathbf{a}, \mathbf{t})$ is continuous in $\mathbf{a}$, there exists a PSNE in nondecreasing strategies. 
Theorem 4 may be especially useful in games with multiplicatively separable payoffs (for example, when $u_{i}(\mathbf{a}, \mathbf{t})=h_{i}(\mathbf{a}) \cdot c_{i}\left(a_{i}, t_{i}\right)$ or $\left.u_{i}(\mathbf{a}, \mathbf{t})=h_{i}\left(\mathbf{a}, t_{i}\right) \cdot c_{i}\left(a_{i}\right)\right)$, since log-supermodularity is preserved by multiplication.

\subsubsection{Noisy Signaling Games}

Our results about existence of PSNE can also be applied to games with alternative timing assumptions. For example, consider a signaling game between two players, where player 1's utility is given by $u_{1}\left(a_{1}, a_{2}, t_{1}\right)$ and player 2 's utility is given by $u_{2}\left(a_{1}, a_{2}\right)$. After observing her type (for example, marginal cost or a parameter of demand), Player 1 takes an action which generates a noisy signal, $t_{2}$, that is observed by player 2 . Player 2 then takes an action. An example is a game of limit pricing (Matthews and Mirman (1983)), where an entrant does not know the cost of the incumbent, but can draw inferences about the incumbent's cost by observing a noisy signal of the incumbent's product market decision (the noise might be due to demand shocks). In another example, Maggi (1999) examines the extent to which noise in the signaling process undermines the first mover advantage in commitment games. Different assumptions about the nature of product market competition lead to different properties of payoffs. Games with strategic substitutes or complements can be analyzed using Theorem 3, and many pricing games are log-supermodular, so that Theorem 4 applies. Otherwise, the following result can be used: ${ }^{15}$ THEOREM 5: Suppose that there are two players, $i=1,2$. Suppose that $(i)$ for $i=1,2, u_{i}(\mathbf{a}, \mathbf{t})$ satisfies weak SCP-IR in $\left(a_{i}, t_{j}\right)$ and $\left(a_{i}, a_{j}\right)$, and is supermodular in $\left(a_{i}, t_{i}\right)$, and (ii) the types are affiliated with non-moving support. Then the game satisfies SCC. Thus, under Al, if either $\mathcal{A}$ is finite, or else for all $i, \mathcal{A}_{i}=\left[a_{i}, \bar{a}_{i}\right]$ and $u_{i}(\mathbf{a}, \mathbf{t})$ is continuous in $\mathbf{a}$, there exists a PSNE in nondecreasing strategies. 


\section{Games With Discontinuities: AuCtions AND PRicing Games}

\subsection{Existence of PSNE}

Auctions and resource allocation games are perhaps the most widely studied applications of games of incomplete information. The problem is to allocate one or more goods to a subset of a group of agents, where each agent has private information about her value for the good. For example, in a first-price auction for a single good, each player submits a sealed bid after observing her type, and the highest bidder receives the good and pays her bid. Corollary 2.1 cannot be applied to auction games, because a player sees a discrete change in her payoffs depending on whether she is a "winner" or a "loser" in the auction. If any opponents use a given bid $b$ with positive probability, a player's probability of winning, and thus expected payoff, changes discontinuously at $b$. Thus, we say that the discontinuity arises as a result of "mass points" in the distribution over opponent actions.

The literature has focused on the existence question primarily for the case of first-price auctions. Two main approaches have been used: (i) establishing that a solution exists to a set of differential equations (Lebrun (1997), Bajari (1997), Lizzeri and Persico (1997)), and (ii) establishing that an equilibrium exists when either types or actions are drawn from finite sets, and then invoking limiting arguments (Lebrun (1996), Maskin and Riley (1999)).

This paper takes the second approach. Theorem 1 implies that in any auction where the SCC is satisfied, a PSNE exists so long as bids must be placed in discrete units (as in many real-world settings). This result holds even if there is a reserve price, or if there are different reserve prices for different bidders.

Thus, any concern about existence of PSNE in auction games with the SCC must focus on discontinuities that arise in the limit as the bidding units become small. Yet, this concern is 
serious: in some examples, there exists a PSNE for every finite action set, but not in the continuum-action case (for example, see Fullerton and McAfee (1999)). Our approach is to use properties of PSNE of finite-action games to show that in the limit, as the action set gets fine, no "mass points" arise and thus payoffs are continuous.

The setup is given as follows. Winners receive payoffs $\bar{v}_{i}\left(a_{i} \mathbf{t}\right)$, while losers receive payoffs $\underline{v}_{i}\left(a_{i}, \mathbf{t}\right) .{ }^{16}$ The allocation rule $\varphi_{i}(\mathbf{a})$ specifies the probability that player $i$ wins as a function of the actions taken by all players. Thus, player $i$ 's payoff given a realization of types and actions is

$$
\begin{aligned}
u_{i}(\mathbf{a}, \mathbf{t}) & =\varphi_{i}(\mathbf{a}) \cdot \bar{v}_{i}\left(a_{i}, \mathbf{t}\right)+\left(1-\varphi_{i}(\mathbf{a})\right) \cdot \underline{v}_{i}\left(a_{i}, \mathbf{t}\right) \\
& =\underline{v}_{i}\left(a_{i}, \mathbf{t}\right)+\varphi_{i}(\mathbf{a}) \cdot \Delta v_{i}\left(a_{i}, \mathbf{t}\right),
\end{aligned}
$$

where $\Delta v_{i}\left(a_{i}, \mathbf{t}\right) \equiv \bar{v}_{i}\left(a_{i}, \mathbf{t}\right)-\underline{v}_{i}\left(a_{i}, \mathbf{t}\right)$. Player $i$ 's expected payoffs can be written

$$
\begin{aligned}
U_{i}\left(a_{i}, t_{i} ; \boldsymbol{\alpha}_{-i}(\cdot)\right) & =\int u_{i}\left(a_{i}, \boldsymbol{\alpha}_{-i}\left(\mathbf{t}_{-i}\right), \mathbf{t}\right) f\left(\mathbf{t}_{-i} \mid t_{i}\right) d \mathbf{t}_{-i} \\
& =\int \underline{v}_{i}\left(a_{i}, \mathbf{t}\right) \cdot f\left(\mathbf{t}_{-i} \mid t_{i}\right) d \mathbf{t}_{-i}+\int \Delta v_{i}\left(a_{i}, \mathbf{t}\right) \cdot \varphi_{i}\left(a_{i}, \boldsymbol{\alpha}_{-i}\left(\mathbf{t}_{-i}\right)\right) \cdot f\left(\mathbf{t}_{-i} \mid t_{i}\right) d \mathbf{t}_{-i} .
\end{aligned}
$$

There are several classes of examples with this structure. In a first-price auction, the winner receives the object and pays her bid, so that $\bar{v}_{i}\left(a_{i}, \mathbf{t}\right)$ is the value of winning at bid $a_{i}$, while losers get payoffs of $\underline{v}_{i}\left(a_{i}, \mathbf{t}\right)=0$. Note that $\Delta v_{i}\left(a_{i}, \mathbf{t}\right)$ can be negative, if the winner pays more than the object is worth. In an all-pay auction, the player pays her bid no matter what, but the winner receives the object. Below, we show that several oligopoly games also fit into the framework.

In most auction models, participation is voluntary: there is some outside option, such as not placing a bid, that provides a fixed, certain utility to the agent, typically normalized to zero. We refer to this action as $Q<\min \left\{\underline{a}_{1}, . ., \underline{a}_{l}\right\}$. The allocation rules take the form

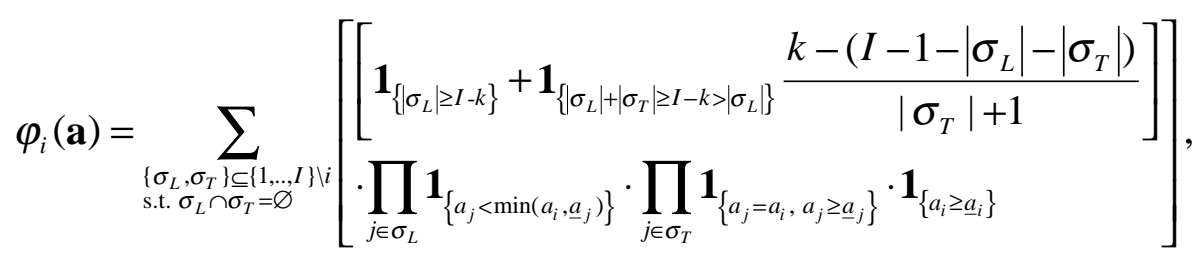


where in this definition, $k$ represents the number of units to be allocated, $\sigma_{L}$ represents a set of opponents defeated by player $i$, and $\sigma_{T}$ represents a set of opponents who tie with player $i$. Thus, player $i$ receives the object with probability zero if $k$ or more opponents choose actions such that $a_{j}>a_{i}$, and with probability 1 if $I-k$ or more opponents choose actions such that $a_{j}<a_{i}$. The remaining events are "ties," resolved randomly. In a first-price auction for a single object, $k=1$, and the player wins with probability zero if 1 or more opponents place a higher bid. All of our results below continue to apply if we consider "biased" allocation rules, ${ }^{17}$ and the rule for resolving ties can also be generalized.

We group together several assumptions.

ASSUMPTION A2: For all $i=1, \ldots, I$, all $a_{i} \in\left[\underline{a}_{i}, \bar{a}_{i}\right]$ and all $\mathbf{t} \in T:(i)$ The support of $F(\mathbf{t})$ is a product set; $\left(\right.$ ii) $\bar{v}_{i}\left(a_{i}, \mathbf{t}\right)$ and $\underline{v}_{i}\left(a_{i}, \mathbf{t}\right)$ are bounded and continuous in $\left(a_{i}, \mathbf{t}\right) ;$ (iii) $\bar{v}_{i}(Q, \mathbf{t})=0$, $\underline{v}_{i}(Q, \mathbf{t})=0$, and $\Delta v_{i}\left(\bar{a}_{i}, \overline{\mathbf{t}}\right)<0$, where $\overline{\mathbf{t}}=\left(\bar{t}_{1}, . ., \overline{t_{I}}\right) ;(i v) \Delta v_{i}\left(a_{i}, \mathbf{t}\right)$ is strictly increasing in $\left(-a_{i}, t_{i}\right) ;$

(v) There exists a $\lambda>0$ such that, for all $\varepsilon>0, \Delta v_{i}\left(a_{i}, \mathbf{t}_{-i}, t_{i}+\varepsilon\right)-\Delta v_{i}\left(a_{i}, \mathbf{t}_{-i}, t_{i}\right) \geq \lambda \varepsilon$.

Part (i) guarantees that the support of $F\left(\mathbf{t}_{-i} \mid t_{i}\right)$ does not vary with $t_{i}$; thus, any action used by a player with positive probability is viewed as having positive probability by all types of all opponents. Part (ii) includes regularity assumptions. Part (iii) normalizes the "outside option" to zero, and further guarantees that the players have available actions larger than any they would choose to use in equilibrium. Part (iv) requires that choosing a higher action decreases the gain from winning, and the gain to winning is strictly increasing in the type. Part (v) requires that there is a uniform lower bound on the slope (with respect to the action) of the gain to winning. All of these assumptions, or more stringent ones, are standard (though often implicit) in the literature on auctions. 
Now consider an existence result for this class of games. We can construct a set of strategies, denoted $\beta^{*}(\cdot)$, as the limit of a sequence of equilibria to finite-action games, $\left\{\beta^{n}(\cdot)\right\}$, as in Theorem 2. Because of the potential discontinuity described above, Theorem 2 cannot be applied directly to establish that $\beta^{*}(\cdot)$ is a PSNE of the continuum-action game. If we can prove that there are no mass points in the limit (that is, no action is used with positive probability), continuity will be restored and the arguments of Theorem 2 can be applied.

Our approach (detailed formally in the Appendix) is to rule out mass points using the fact that $\left\{\beta^{n}(\cdot)\right\}$ converges to $\beta^{*}(\cdot)$ uniformly except on a set of arbitrarily small measure, together with the fact that we can characterize certain properties of each $\beta^{n}(\cdot)$ based on the fact that $\beta^{n}(\cdot)$ is a PSNE. The argument can be sketched as follows. We proceed by contradiction. Suppose $\beta^{*}(\cdot)$ specifies that two players, $i$ and $j$, both use action $b$ with positive probability (which cannot be an equilibrium by standard arguments). Let $S_{i}$ be the subset of player $i$ 's types that use $b$ under $\beta^{*}(\cdot)$, and likewise for player $j$. Consider the requirements placed on $\left\{\beta^{n}(\cdot)\right\}$. Given a $d>0$, for some $n$ large enough (i.e., some action grid fine enough), $\boldsymbol{\beta}^{n}(\cdot)$ must specify that all but a small subset of player $i$ 's types in $S_{i}$ use an action on $(b-d, b+d)$, and likewise for player $j$. But then we will argue that there must be some action grid fine enough, and some $d$ small enough, such that the "cost" to player $j$ of increasing her action by $d$ is less than the benefit from the increase in the probability of defeating most of the types $t_{i} \in S_{i}$. In this case, for player $j$, almost all types $t_{j} \in S_{j}$ prefer $b+d$ to any action on $[b-d, b+d]$, contradicting uniform convergence to $\beta^{*}(\cdot)$.

To complete the latter argument, it is necessary to establish that increasing the probability of winning (for example, by increasing the action from $b-d$ to $b+d$ ) is in fact a benefit for most of the player types in $S_{i}$ and $S_{j}$. That requires two building blocks.

The first building block guarantees that player $i$ 's expected gain from winning goes up when 
player $i$ conditions on winning with a higher action. We require the following additional notation: let $W_{i}\left(a_{i} ; \boldsymbol{\alpha}_{-i}\right)$ denote the event that the realization of $\mathbf{t}_{-i}$ and the outcome of the tiebreaking mechanism are such that player $i$ wins with $a_{i}$, when opponents use strategies $\alpha_{-i}$. Thus, $\operatorname{Pr}\left(W_{i}\left(a_{i} ; \boldsymbol{\alpha}_{-i}\right)=1 \mid t_{i}\right)=\int \varphi_{i}\left(a_{i}, \boldsymbol{\alpha}_{-i}\left(\mathbf{t}_{-i}\right)\right) \cdot f\left(\mathbf{t}_{-i} \mid t_{i}\right) d \mathbf{t}_{-i}$.

ASSUMPTION A3: For all $i=1, . ., I$, all $a_{i}, a_{i}^{\prime} \in\left[\underline{a}_{i}, \bar{a}_{i}\right]$, and whenever every opponent $j \neq i$ uses $a$ strategy $\alpha_{j}:\left[t_{-j}, \bar{t}_{j}\right] \rightarrow \mathscr{A}_{j}$ that is nondecreasing, $E\left[\Delta v_{i}\left(a_{i}, \mathbf{t}\right) \mid t_{i}, W_{i}\left(a_{i}^{\prime} ; \alpha_{-i}\right)\right]$ is strictly increasing in $t_{i}$ and nondecreasing in $a_{i}^{\prime}$.

This assumption is standard in the literature, but it is fairly restrictive. Although it holds trivially for private value auctions, it requires strong assumptions on the type distribution in more general models. For first-price auctions, Milgrom and Weber (1982) showed that when $\Delta v_{i}$ is nondecreasing in $\mathbf{t}$ and strictly increasing in $t_{i}$, A3 holds if the types are affiliated.

The second building block establishes that if $b-d$ is a best response for type $t_{i}$ of player $i$, this type prefers winning to losing at $b+d$. This is not trivial, because player $i$ might win with probability 0 using action $b-d$, in which case nothing about her preferences for winning at $b-d$ can be inferred. To see a problematic example, consider a single-unit, first-price auction, and a sequence of minimum bid increments $\varepsilon_{n} \rightarrow 0$. Suppose that for each $n$, in the PSNE of the corresponding finite-action game, a set $S_{i}$ of player $i$ types uses action $b-\varepsilon_{n}$ and a set $\left[t_{-j}, t_{j}^{\prime}\right]$ of player $j$ types uses action $b$. Thus, $b-\varepsilon_{n}$ wins with probability zero for player $i$ for each $n$, and it is possible that losing is preferred to winning by player $i$ 's types in $S_{i}$. In this case, even though winning at $b$ is not desirable for these types, the limiting strategies require them to choose $b$ and win with positive probability.

To this rule out, in the Appendix we show that for every finite-action game, there exists a 
PSNE that is robust to perturbations that generate a small probability that each action wins.

LEMMA 4: For $i=1, . ., I$, let $\mathcal{A}_{i}$ be finite, with $Q \in \mathcal{A}_{i}$. Consider an auction game with payoffs given by (2)-(3), and assume that Al and the SCC hold. (i) For all $\delta>0$, there exists a PSNE in nondecreasing strategies of the modified game, denoted $\hat{\boldsymbol{\beta}}(\cdot ; \delta)$, where each player type on $t_{i} \in\left[t_{-i}, t_{-i}+\delta\right]$ is required to use action $Q$. (ii) There exists a PSNE in nondecreasing strategies, $\beta^{*}(\cdot)$, such that: $\beta^{*}(\cdot)=\lim _{k \rightarrow \infty} \hat{\beta}\left(\cdot ; \delta_{k}\right)$ for some sequence $\left\{\delta_{k}\right\}$, where $\lim _{k \rightarrow \infty} \delta_{k}=0$ and $\lim _{k \rightarrow \infty} E\left[\Delta v_{i}\left(\hat{\beta}_{i}\left(t_{i} ; \boldsymbol{\delta}_{k}\right), \mathbf{t}\right) \mid t_{i}, W_{i}\left(\hat{\beta}_{i}\left(t_{i} ; \boldsymbol{\delta}_{k}\right) ; \hat{\beta}_{-i}\left(\cdot ; \boldsymbol{\delta}_{k}\right)\right)\right] \geq 0$ for all $t_{i} \in T_{i} \cdot$

With these two building blocks in place, we can rule out the possibility that two players both use the same action with positive probability. Another case of potential concern occurs if just one player, player $i$, uses action $b$ with positive probability. Although this case can be ruled out using somewhat more involved arguments, we will not undertake this exercise here because it does not affect existence of PSNE: only a countable number of actions can be used by player $i$ with positive probability, and thus the set of opponent types who see a discontinuity in payoffs has measure zero. Since Bayesian Nash equilibrium only requires that almost every type chooses an optimal action, such mass points do not affect existence. The conclusion then follows:

THEOREM 6: For all $i$, let $\mathscr{A}_{i}=Q \cup\left[\underline{a}_{i}, \bar{a}_{i}\right]$. Consider an auction game with payoffs described by (2)-(3), and assume A1-A3, and that the game satisfies the SCC. Then, there exists a PSNE in nondecreasing strategies.

Theorem 6 generalizes the best available existence results about first-price auctions, multiunit pay-your-bid auctions where agents have a unit demand, and all-pay auctions. It allows for bidder-specific reserve prices, heterogeneous agents, general risk preferences, and correlated type distributions-so long as the SCC holds. Before discussing the primitive conditions under which 
the SCC holds, we consider some further implications of the theorem.

First, once existence is established for the continuous-action game, standard arguments can be used to verify the usual regularity properties (including optimality of actions for every type). For example, strategies are strictly increasing on the interior of the set of actions played with positive probability. Further, with appropriate differentiability assumptions, a differential equations approach can be used for characterizations.

Second, Theorems 2 and 6 not only provide existence results for the continuum case; they also establish that finite-action games can be used to closely approximate continuum-action games. Thus, the continuum model is an appropriate abstraction for auctions in which fixed bid increments are small. As well, revenue and allocation in an auction will not be very sensitive to small changes in the number of bid increments allowed.

Perhaps more importantly, the convergence results also motivate a computational algorithm. This may be particularly useful in the case of first-price auctions, since in the absence of general characterization theorems and functional form examples, computation of equilibria to first-price auctions is the main tool available to evaluate the effects of mergers (or collusion) between bidders in auctions. Prior to Marshall et al (1994), there were no general numerical algorithms available for computing equilibria to asymmetric first-price auctions. Numerical computation of equilibria in asymmetric first-price auctions in the independent private values case is difficult due to pathological behavior of the system of differential equations at the origin. Marshall et al (1994) provide computations for the independent private values case for a particular functional form; see also Bajari (1997).

Theorem 6 suggests an alternative: compute equilibria to games with successively finer action sets. The computation of a finite-action equilibrium requires searching for a fixed point to 
the correspondence $\Gamma$ defined in Section 2, where the calculation of $\Gamma(\mathbf{X})$ is a simple exercise of calculating the best-response jump points for each player. The more difficult part of the problem is solving the nonlinear set of equations $\mathbf{X}=\Gamma(\mathbf{X})$. There are a number of standard ways to approach this problem. There is not a global "contraction mapping" theorem, and so the simplest algorithm $\mathbf{X}^{k+1}=\Gamma\left(\mathbf{X}^{k}\right)$ is not guaranteed to converge, and indeed it does not appear to in numerical trials. The working paper (Athey, 1997) provides a number of computational examples which could be computed using either variations on the algorithm $\left.\mathbf{X}^{k+1}=\lambda \cdot \Gamma\left(\mathbf{X}^{k}\right)+(1-\lambda) \cdot \mathbf{X}^{k}\right)$ ), or quasiNewton approaches. ${ }^{18}$

\subsection{Characterizing the Single Crossing Condition in Auctions and Pricing Games}

This section characterizes properties of primitives (utility functions and type distributions) that imply the SCC and A3 for a variety of widely-studied auctions and pricing games. To begin, we formally describe the auction environments of interest. For many auction models, we can model a bidder's utility from obtaining the object as $V_{i}\left(Z_{i}-m\right)$, where $Z_{i}$ is the value of the object and $m$ is the payment to the auctioneer. The utility from not receiving the object, and making payment $m$, is $V_{i}(-m)$. Normalize $V_{i}(0)=0$. Further assume that $V_{i}$ is strictly increasing with nonvanishing slope. A bidder is risk-neutral if $V_{i}$ is linear, risk-averse if $V_{i}$ is concave, and "not too risk-loving" if $\ln \left(V_{i}\right)$ is concave.

In a first-price auction, as well as in a multi-unit auction where each bidder has unit demand and pays her bid if she receives a unit, ${ }^{19} \underline{v}_{i}\left(a_{i}, \mathbf{t}\right)=0$. All-pay auctions have been used to model activities such as lobbying (Becker (1983), Baye, Kovenock, and de Vries (1993)) and arms races (O'Neill, 1986); in this model, $\underline{v}_{i}\left(a_{i}, \mathbf{t}\right)=V_{i}\left(-a_{i}\right)$.

Consider four alternative sets of assumptions for the auction models. In the general affiliated values setting, $\bar{v}_{i}\left(a_{i}, \mathbf{t}\right)$ is nondecreasing in $\mathbf{t}$, supermodular in $\left(a_{i}, t_{i}\right)$, and types are affiliated. In an 
affiliated private values setting, $Z_{i}=t_{i}$, so that $\bar{v}_{i}\left(a_{i}, \mathbf{t}\right)=V_{i}\left(t_{i}-a_{i}\right)$, and types are (weakly) affiliated; independent private values is then a special case. In the conditionally independent common values model, $Z_{i}=Z$ for all $i$, bidders see signals $s_{i}=Z+\varepsilon_{i}$, and the $\varepsilon_{i}$ are distributed independently across bidders, conditional on $Z$. This special case is commonly used in applications, including the recent empirical literature on auctions (see, e.g., Li, Perrigne, and Vuong, 1999). In the positive value interdependence, independent information model, the bidders types are distributed independently. The bidder's utility from an allocation, $\bar{v}_{i}\left(a_{i}, \mathbf{t}\right)$, is nondecreasing in the opponent types, and further, bidder $i$ 's utility is supermodular in $\left(a_{i}, t_{i}\right)$ and in $\left(t_{i}, t_{j}\right)$ for all opponents $j$. These assumptions hold, for example, if the bidder is risk neutral and $Z_{i}$ is a weighted sum or average of opponent types.

Now consider oligopoly games. In the Bertrand pricing game, the firm's payoff from serving the market is given by $\left(p-c_{i}\right) D(p)$, where $D$ is nonincreasing, $p$ is the sale price, and $c_{i}$ is the marginal cost of production, taken to be incomplete information. The seller serves the market if she has the lowest price, shares it in the event of a tie, and earns zero profit otherwise. Spulber (1995) uses such a model, with private values model and symmetric firms, to show that incomplete information can lead to above-marginal-cost pricing; Athey, Bagwell, and Sanchirico (1998) use the same model as the stage game in a repeated-game study of price rigidity. The model also applies to procurement auction settings, where the bid is a per-unit price, and the buyer's demand decreases with the winning price. Placing the model in our framework, $\underline{v}_{i}\left(a_{i}, \mathbf{t}\right)=0$. In a private values setting, $\bar{v}_{i}\left(a_{i}, \mathbf{t}\right)=\left(-a_{i}+t_{i}\right) D\left(-a_{i}\right)$, where $-a_{i}$ is the price paid and $-t_{i}$ is the marginal cost of production. Although the private value setting is appropriate for capturing idiosyncratic productivity differences, firms might face common uncertainty about input prices, or they might engage in research and development with spillovers. Then the general affiliated 
values setting may be applicable, where $\bar{v}_{i}\left(a_{i}, \mathbf{t}\right)=E\left[\left(-a_{i}+Z_{i}\right) D\left(-a_{i}\right) \mid \mathbf{t}\right]$. The conditionally independent common values case is analogous to the first-price auction; the positive value interdependence, independent information case has $Z_{i}=g_{i}(\mathbf{t})$, where $g_{i}$ is nondecreasing and supermodular in $\left(t_{i}, t_{j}\right)$ for $i \neq j$.

A final class of models was introduced by Varian (1980) to study price dispersion. Firms sell to two types of consumers, a set $C$ of "captured" consumers with inelastic demand, and a set $S$ of price-sensitive consumers who buy from the firm with the lowest price. Varian (1980) derives a mixed-strategy equilibrium to this model, and interprets the model as a theory of sales. Bagwell and Wolinsky (1998) analyze an extension where firms have incomplete information about marginal cost. To analyze the model our framework, we let $\underline{v}_{i}\left(a_{i}, \mathbf{t}\right)=\left(-a_{i}+t_{i}\right) D^{C}\left(-a_{i}\right)$ and $\bar{v}_{i}\left(a_{i}, \mathbf{t}\right)=\left(-a_{i}+t_{i}\right)\left(D^{C}\left(-a_{i}\right)+D^{S}\left(-a_{i}\right)\right)$ for the case of private values; the other informational settings are constructed in a similar manner.

The following result establishes existence of PSNE in these games.

THEOREM 7: The SCC and A3 are satisfied in the following pricing games and auction environments (where in all cases, players are potentially heterogeneous, and bidder-specific reserve prices are permitted):

1. First-price auction. The setting is either: (i) affiliated private values, bidders are not too risk loving; (ii) positive value interdependence, independent information; (iii) conditionally independent common values, and bidders are (weakly) risk averse; or (iv) general affiliated values, but only two bidders.

2. Multi-unit discriminatory auction where each bidder demands a single unit. The setting is independent private values; bidders are not too risk-loving.

3. All-pay auction. The setting is either (i) independent private values, (weakly) risk averse 
bidders; or (ii) positive value interdependence, independent information.

4. Bertrand pricing game with downward-sloping demand. The setting is either: (i) affiliated private values; (ii) positive value interdependence, independent information; (iii) conditionally independent common values; or (iv) general affiliated values, but only two bidders.

5. Price dispersion model. The setting is either (i) independent private values; or (ii) positive value interdependence, independent information.

Thus, under A1-A2, if participation is voluntary and either $A$ is finite, or else for each $i$, bids must lie on $\left[a_{i}, \bar{a}_{i}\right]$, a PSNE exists in nondecreasing strategies.

The proof of this result is in the Appendix. We highlight the proof for a few special cases where the SCC is especially easy to check. Consider the independent private values information structure. Then, in the first-price auction, payoffs to a bidder are given by $V_{i}\left(t_{i}-a_{i}\right) \operatorname{Pr}\left(a_{i}\right.$ wins $)$. It is immediate to verify that this function is log-supermodular, no matter what strategies are used by opponents, if and only if $V_{i}$ is log-concave. Then, the SCC holds. In the all-pay auction, payoffs are given by $\left(V_{i}\left(t_{i}-a_{i}\right)-V_{i}\left(-a_{i}\right)\right) \operatorname{Pr}\left(a_{i}\right.$ wins $)-V_{i}\left(-a_{i}\right)$. No matter what opponents do, this expression is supermodular so long as $V_{i}$ is increasing and concave, and again the SCC holds. The oligopoly games are similarly transparent.

In each of the games described above, Theorem 7 extends the set of environments where existence of PSNE is known. Both of the pricing games have been analyzed only for symmetric bidders and independent, private values. For first-price auctions, Lebrun (1997) establishes existence for the independent private values case, where the value distributions have a common support, and there is a single reserve price for all bidders. In contrast, our result allows the value distributions of different bidders to have different supports, and it further allows for bidder- 
specific reserve prices, as would maximize revenue in an auction with heterogeneous bidders. Maskin and Riley (1999) establish existence under the affiliated private values and positive value interdependence, independent types settings; they do not consider reserve prices or the conditionally independent common values cases, settings that are especially relevant for empirical applications (for example, U.S. off-shore oil lease auctions have reserve prices and heterogeneous bidders).

Amann and Leininger (1996) studied asymmetric all-pay auctions for the two-bidder, private values case. In independent research, Lizzeri and Persico (1997) analyze two-bidder auctions, establishing existence and uniqueness of equilibria in a general class of auction games that includes first-price and all-pay auctions. Their approach does not extend to more than two bidders unless bidders are symmetric, and while they include reserve prices, each bidder must face the same reserve price. For the general affiliated values model of all-pay auctions, Krishna and Morgan (1997) establish existence for the case of symmetric, risk-neutral bidders. Even in that special case, additional assumptions on the type distribution are required. Their approach can potentially be generalized to heterogeneous bidders, but this is left for future work.

It should be emphasized that neither the existing literature, nor Theorem 7, provides sufficient conditions for existence in the general affiliated values model of first-price auctions, with more than two heterogeneous bidders; as discussed in Athey (1997) and Athey (1998b), competing effects may arise in that case. However, Theorem 6 does show that if primitive conditions (even functional forms) can be found whereby the SCC does hold for the general case (i.e., if the competing effects are assumed to be small, as in an extension of the conditionally independent common values model where a "small" amount of correlation is permitted between the errors $\varepsilon_{i}$ ), existence of PSNE follows immediately; non-monotonicity of bidding functions is 
the only concern in the more general model.

None of the above authors focus on multi-unit auctions. Indeed, extending the results about multi-unit auctions to allow for more general primitive assumptions, and multi-unit demands, represents an interesting avenue for future research.

\section{CONCLUSIONS}

This paper has introduced a restriction called the single crossing condition (SCC) for games of incomplete information. We have shown that when the SCC is satisfied, PSNE exist when the set of available actions is finite. Further, with appropriate continuity or in auction games, there exists a sequence of equilibria of finite-action games that converges to an equilibrium in a game with a continuum of actions.

The results developed in this paper have the following implications. First, existence of PSNE in nondecreasing strategies can be verified by checking economically interpretable conditions, conditions which are satisfied by construction in many economic applications. Second, in applications the question of existence has been reduced to the simpler question of comparative statics in a single-agent decision problem. As shown in Sections 3.2 and 4.2 (see also Athey (1998a, b)), a variety of tools are available to solve this comparative statics problem. Finally, the constructive approach to existence taken in this paper has advantages for numerical computation. The equilibria are straightforward to calculate for finite-action games, and they approximate the equilibria of continuous-action games.

Massachusetts Institute of Technology, Department of Economics, E52-252C, 50 Memorial

Drive, Cambridge, MA 02142-1347,USA; athey@mit.edu, http://web.mit.edu/athey/www/. 


\section{APPENDIX}

PROOF OF LEMMA 4: (i) Define the constrained best response of player $i$ to an arbitrary (constrained or unconstrained) strategy by opponents represented by $\mathbf{X}: \hat{a}_{i}^{B R}\left(t_{i} \mid \mathbf{X}, \boldsymbol{\delta}\right)$ is set equal to $a_{i}^{B R}\left(t_{i} \mid \mathbf{X}\right)$ when $t_{i}>\underline{t}_{i}+\delta$, to $\left\{Q \cup a_{i}^{B R}\left(t_{i} \mid \mathbf{X}\right)\right\}$ when $t_{i}=\underline{t}_{i}+\delta$, and $\{Q\}$ when $t_{i}<t_{i}+\delta$.

Using this notation, we modify our correspondence, as follows:

$\hat{\Gamma}_{i}(\mathbf{X}, \delta)=\left\{\mathbf{y} \in \Sigma^{M}: \exists \alpha_{i}(\cdot)\right.$ which is consistent with $\mathbf{y}$ such that $\left.\forall t_{i} \in T_{i}, \alpha_{i}\left(t_{i}\right) \in \hat{a}_{i}^{B R}\left(t_{i} \mid \mathbf{X}, \delta\right)\right\}$.

The arguments of Lemma 3 can be extended in a straightforward way to derive existence of a fixed point of $\hat{\Gamma}(\cdot, \delta)$ for all $\delta>0$; details can be found in the working paper (Athey, 1997). Then, consider a sequence $\mathbf{X}^{k}$ such that $\mathbf{X}^{k} \in \hat{\Gamma}\left(\mathbf{X}^{k}, 1 / k\right)$ for each $k$. Since each $\mathbf{X}^{k}$ is an element of a compact subset of finite-dimensional Euclidean space, we can find a subsequence $\{k\}$ such that $\left\{\mathbf{X}^{k}\right\}$ converges to a matrix $\mathbf{X}$, and we simply need to establish that $\mathbf{X} \in \Gamma(\mathbf{X})$. Consider $t_{i}$ such that $t_{i} \in T_{i} \backslash\left\{\mathbf{x}^{i}\right\}$. Then there exists an $m \in\{0, . ., M\}$ such that $x_{m}^{i}<t_{i}<x_{m+1}^{i}$. Since $\mathbf{x}^{i, k}$ converges to $\mathbf{x}^{i}$, there must exist an $K$ such that, for all $k>K, x_{m}^{i, k}<t_{i}<x_{m+1}^{i, k}$ and $t_{i}>t_{i}+1 / k$. Find such a $k>K$. Then $A_{m} \in \hat{a}_{i}^{B R}\left(t_{i} \mid \mathbf{X}^{k}, 1 / k\right)$ since $\mathbf{X}^{k} \in \Gamma\left(\mathbf{X}^{k}\right)$. By definition and since $t_{i}>t_{i}+1 / k, A_{m} \in a_{i}^{B R}\left(t_{i} \mid \mathbf{X}^{k}\right)$. But, since $V_{i}\left(a_{i} ; \mathbf{X}, t_{i}\right)$ is continuous in $\mathbf{X}$, if $V_{i}\left(A_{m} ; \mathbf{X}^{k}, t_{i}\right) \geq V_{i}\left(A_{m^{\prime}} ; \mathbf{X}^{k}, t_{i}\right)$ for all $k>K$ and all $m^{\prime}$, then $V_{i}\left(A_{m} ; \mathbf{X}, t_{i}\right) \geq V_{i}\left(A_{m^{\prime}} ; \mathbf{X}, t_{i}\right)$. This implies $A_{m} \in a_{i}^{B R}\left(t_{i} \mid \mathbf{X}\right)$, as desired. (ii) Finally, observe that for each $k$, each action greater than $Q$ wins with positive probability. Since $Q$ is an available action that yields zero payoffs, by revealed preference, $E\left[\Delta v_{i}\left(\hat{\beta}_{i, n}\left(t_{i} ; \boldsymbol{\delta}_{k}\right), \mathbf{t}\right) \mid t_{i}, W_{i}\left(\hat{\beta}_{i, n}\left(t_{i} ; \boldsymbol{\delta}_{k}\right) ; \hat{\beta}_{n}\left(\cdot ; \boldsymbol{\delta}_{k}\right)\right)\right] \geq 0$

for all $k$. Since payoffs are continuous in $\delta_{k}$, the limit as $\delta_{k}$ approaches zero exists. Q.E.D. LEMMA 5: Consider an auction game satisfying A1-A3, (2)-(3), and the SCC. Let $\boldsymbol{\beta}^{*}(\cdot)$ be the limit of a convergent subsequence of equilibrium strategies to finite games, $\boldsymbol{\beta}^{n}(\cdot)$, that satisfy 
Lemma 4 (ii). Then the following holds:

$$
\text { For all } i \neq j \text { and all } b>Q, \operatorname{Pr}\left(\beta_{i}^{*}\left(t_{i}\right)=b\right) \cdot \operatorname{Pr}\left(\beta_{j}^{*}\left(t_{j}\right)=b\right)=0 .
$$

PROOF: To begin we introduce some notation for the event that, when player $i$ 's opponents use strategies $\beta_{-i}^{*}(\cdot)$, the realization of $\mathbf{t}_{-i}$ and the outcome of the tie-breaking mechanism are such that the action $a_{i}$ produces the stated outcome:

$W_{i}^{*}\left(a_{i}\right)$ : Player $i$ wins using $a_{i}$ (either by winning a tie or winning outright). $\tau_{i}^{W^{*}}\left(a_{i}\right), \tau_{i}^{L^{*}}\left(a_{i}\right)$ : Player $i$ ties for winner at $a_{i}$ and player $i$ wins (resp. loses) the tie. Further, let $W_{i}^{n}\left(a_{i}\right)$ be the event that player $i$ wins using $a_{i}$ when opponents use strategies $\beta_{-i}^{n}(\cdot)$. For the case where $W_{i}^{n}\left(\beta_{i}^{n}\left(t_{i}\right)\right)$ occurs with probability 0 , we use the following convention:

$$
E\left[\Delta v_{i}\left(\beta_{i}^{n}\left(t_{i}\right), \mathbf{t}\right) \mid t_{i}, W_{i}^{n}\left(\beta_{i}^{n}\left(t_{i}\right)\right)\right]=\lim _{k \rightarrow \infty} E\left[\Delta v_{i}\left(\hat{\beta}_{i}^{n}\left(t_{i} ; \boldsymbol{\delta}_{k}\right), \mathbf{t}\right) \mid t_{i}, \hat{W}_{i}^{n}\left(\hat{\beta}_{i}^{n}\left(t_{i} ; \boldsymbol{\delta}_{k}\right) ; \boldsymbol{\delta}_{k}\right)\right],
$$

where $\delta_{k} \rightarrow 0, \hat{\boldsymbol{\beta}}^{n}\left(\cdot ; \delta_{k}\right)$ is defined in Lemma 4 , and $\hat{W}_{i}^{n}\left(a_{i} ; \delta_{k}\right)$ is the event that player $i$ wins with $a_{i}$ when opponents use strategies $\hat{\boldsymbol{\beta}}_{-i}^{n}\left(\cdot ; \delta_{k}\right)$.

We proceed by contradiction. Assume (4) fails for some $b$. Let $K=\left\{i: \operatorname{Pr}\left(\beta_{i}^{*}\left(t_{i}\right)=b\right)>0\right\}$ (note $|K| \geq 2$ ), and let $J=\{1, \ldots, I\} \backslash K$. Recall that for each $n, \beta_{i}^{n}(\cdot)$ is measurable, and the sequence converges to $\beta_{i}^{*}(\cdot)$; thus the sequence converges uniformly to $\beta_{i}^{*}(\cdot)$ except on a set of arbitrarily small measure (Royden, 1988, p. 73).

A little notation. Let $\varepsilon_{n}$ be the minimum increment to actions (assumed for simplicity to be the same for all players and independent of the level of the action) for the action set $\mathscr{A}^{2}$. Find a set of types $\mathbf{E}$ such that $\beta_{n}(\cdot)$ converges uniformly to $\beta^{*}(\cdot)$ except for types $\mathbf{t} \in \mathbf{E}$, and such that for all $i \in K$,

$$
\gamma_{i} \equiv \inf _{t_{i}}\left(\operatorname{Pr}\left(\tau_{i}^{L^{*}}(b) \mid t_{i}\right)-\operatorname{Pr}\left(\exists j \neq i \text { s.t. } t_{j} \in E_{j} \mid t_{i}\right)\right)>10 \cdot \sup _{t_{i}}\left(\operatorname{Pr}\left(\exists j \neq i \text { s.t. } t_{j} \in E_{j} \mid t_{i}\right)\right) .
$$


For $i \in K$, let $S_{i}=\left\{t_{i}: \beta_{i}^{*}\left(t_{i}\right)=b\right\}$, let $\tilde{S}_{i}=S_{i} \backslash E_{i}$, and let $\tilde{t}_{i}=\inf \tilde{S}_{i}$.

For any $d>0$, define $N_{d}$ as the smallest positive integer such that, for all $n>N_{d}$, (i) $b-d>\varepsilon_{n}$ and (ii) for all $i \in K$ and all $t_{j} \in \tilde{S}_{j},\left|\beta_{i}^{n}\left(t_{i}\right)-\beta_{i}^{*}\left(t_{i}\right)\right|<d$.

We consider two claims in turn.

Claim 1: There exists $\psi \in\left(0, \min _{i \in K}\left(\sup \left(\tilde{S}_{i}\right)-\tilde{t}_{i}\right) / 2\right), d>0$, and a positive integer $n>N_{d}$ such that for all $t_{i} \in S_{i}$ such that $t_{i} \geq \tilde{t}_{i}+\psi$, and all $i \in K$, equations (6)-(9) hold when $a^{\prime}=b-d+\varepsilon_{n}$.

$$
\begin{gathered}
\operatorname{Pr}\left(\exists j \in J \text { s.t. } \beta_{j}^{n}\left(t_{j}\right) \in(b-d, b+d) \mid t_{i}\right) \\
+\operatorname{Pr}\left(\exists k \in K, k \neq i, \text { s.t. } \inf \left\{t_{k}^{\prime}: \beta_{k}^{n}\left(t_{k}^{\prime}\right)>b-d\right\}<t_{k}<\tilde{t}_{k}+\psi \mid t_{i}\right)<\frac{1}{2} \gamma_{i} \\
\max _{a^{\prime \prime} \in\left\{b-d+\varepsilon_{n}, ., b+d-\varepsilon_{n}\right\}}\left[\begin{array}{l}
\int\left[\underline{v}_{i}\left(a^{\prime \prime}, \mathbf{t}\right)-\underline{v}_{i}(b+d, \mathbf{t})\right] \cdot f\left(\mathbf{t}_{-i} \mid t_{i}\right) d \mathbf{t}_{-i} \\
\left.+E\left[\Delta v_{i}\left(a^{\prime \prime}, \mathbf{t}\right)-\Delta v_{i}(b+d, \mathbf{t}) \mid t_{i}, W_{i}^{n}\left(a^{\prime \prime}\right)\right] \cdot \operatorname{Pr}\left(W_{i}^{n}\left(a^{\prime \prime}\right) \mid t_{i}\right)\right]<\frac{1}{4} \cdot\left(\frac{\lambda \psi}{2} \gamma_{i}\right) \\
\operatorname{Pr}\left(W_{i}^{n}(b+d) \backslash W_{i}^{n}\left(a^{\prime}\right) \mid t_{i}\right)>\inf \left(\operatorname{Pr}\left(\tau_{i}^{L_{i}^{*} \in S_{i}}(b) \mid t_{i}\right)-\operatorname{Pr}\left(\exists j \neq i \text { s.t. } t_{j} \in E_{j} \mid t_{i}\right)\right) \equiv \gamma_{i} \\
E\left[\Delta v_{i}(b+d, \mathbf{t}) \mid t_{i}, W_{i}^{n}(b+d) \backslash W_{i}^{n}\left(a^{\prime}\right)\right]>\frac{\lambda \psi}{2}
\end{array}\right.
\end{gathered}
$$

Proof of Claim 1: Consider first (6). The first term on the left-hand side is less than $\frac{1}{10} \gamma_{i}$ for small enough $d$ and large enough $n$, by (5) and since $\operatorname{Pr}\left(\beta_{j}^{*}\left(t_{j}\right)=b\right)=0 \forall j=J$ by assumption. Now

consider the second term. By (5), $\sup _{t_{i} \in S_{i}} \operatorname{Pr}\left(\exists k \in K, k \neq i\right.$, s.t. inf $\left.S_{k}<t_{k}<\tilde{t}_{k} \mid t_{i}\right)<\frac{1}{10} \gamma_{i}$. Then, for $\psi$ small enough, $d$ small enough, and $n$ large enough, the second term is less than $\frac{1}{10} \gamma_{i}$, and (6) holds. Pick such $\psi^{\prime}, d^{\prime}$, and $n^{\prime}$. Equation (6) will continue to hold for $0<d<d^{\prime}$ and $n>n^{\prime}$.

For (7), find $0<d^{\prime \prime}<d^{\prime}$ and a corresponding $n^{\prime \prime}>\max \left(n^{\prime}, N_{d^{\prime \prime}}\right)$ such that both (6) and (7) hold when $\psi=\psi^{\prime}$. This is possible because the term in brackets in (7) approaches zero as $d$ approaches 
zero, since $\underline{v}_{i}$ and $\Delta v_{i}$ are continuous.

Now consider equation (8). All opponent types outside $\mathbf{E}_{-i}$ who choose action $b$ in the limit must choose actions on $\left[b-d+\varepsilon_{n}, b+d\right)$ for $n>N_{d}$. By choosing action $b+d$ rather than $b-d+\varepsilon_{n}$, player $i$ chooses a strictly higher action than those types she would lose to or tie with using action $b-d+\varepsilon_{n}$; at worst, all of the types outside $\mathbf{E}_{-i}$ who choose action $b$ in the limit choose action $b-d+\varepsilon_{n}$ with grid $n$, and player $i$ defeats those players she would have otherwise tied with by increasing her action to $b+d$. Thus, (8) holds with $n=n^{\prime \prime}$ and $d=d^{\prime \prime}$.

Finally, consider (9). Let $t_{i}^{\prime}=\tilde{t}_{i}+\psi / 2 \in \tilde{S}_{i}$. Then for $n>N_{d}$, $E\left[\Delta v_{i}\left(\beta_{i}^{n}\left(t_{i}^{\prime}\right), t_{i}^{\prime}, \mathbf{t}_{-i}\right) \mid t_{i}^{\prime}, W_{i}^{n}(b+d)\right] \geq 0$, by Lemma 4 and since winning with $b+d$ rather than the lower, assigned action $\beta_{i}^{n}\left(t_{i}^{\prime}\right)$, increases payoffs by A3. Combining this inequality with the lower bound on the slope of $\Delta v_{i}, \lambda$, and the fact that $t_{i}>\tilde{t}_{i}+\psi$ yields $E\left[\Delta v_{i}\left(\beta_{i}^{n}\left(t_{i}^{\prime}\right), t_{i}, \mathbf{t}_{-i}\right) \mid t_{i}, W_{i}^{n}(b+d)\right]>\lambda \psi / 2$. But, since $\Delta v_{i}$ is continuous in actions, it is possible to find $0<d^{\prime \prime \prime}<d^{\prime \prime}$ and $n^{\prime \prime \prime}>\max \left(n^{\prime \prime}, N_{d^{\prime \prime}}\right)$ such that $E\left[\Delta v_{i}\left(b+d, t_{i}, \mathbf{t}_{-i}\right) \mid t_{i}, W_{i}^{n}(b+d)\right]>\lambda \psi / 2$.

Finally, winning with $b+d$ but not with $b-d+\varepsilon_{n}$ gives higher expected payoffs than winning with $b+d$ by A3. Thus, (6)-(9) hold with $n=n^{\prime \prime \prime}$ and $d=d^{\prime \prime \prime}$.

Claim 2: There exists $\psi \in\left(0, \min _{i \in K}\left(\sup \left(\tilde{S}_{i}\right)-\tilde{t}_{i}\right) / 2\right), d>0$ and a positive integer $n>N_{d}$ such that for all $t_{i} \in S_{i}$ such that $t_{i} \geq \tilde{t}_{i}+\psi$, and all $i \in K, \beta_{i}^{n}\left(t_{i}\right) \notin(b-d, b+d)$.

Proof of Claim 2: Find the parameters guaranteed to exist in Claim 1. Consider $i \in K$. Type $t_{i}$ prefers action $b+d$ to $a^{\prime}$ if: 


$$
\begin{aligned}
& E\left[\Delta v_{i}(b+d, \mathbf{t}) \mid t_{i}, W_{i}^{n}(b+d) \backslash W_{i}^{n}\left(a^{\prime}\right)\right] \cdot \operatorname{Pr}\left(W_{i}^{n}(b+d) \backslash W_{i}^{n}\left(a^{\prime}\right) \mid t_{i}\right) \\
& >\int\left[\underline{v}_{i}\left(a^{\prime}, \mathbf{t}\right)-\underline{v}_{i}(b+d, \mathbf{t})\right] \cdot f\left(\mathbf{t}_{-i} \mid t_{i}\right) d \mathbf{t}_{-i} \\
& \quad+E\left[\Delta v_{i}\left(a^{\prime}, \mathbf{t}\right)-\Delta v_{i}(b+d, \mathbf{t}) \mid t_{i}, W_{i}^{n}\left(a^{\prime}\right)\right] \cdot \operatorname{Pr}\left(W_{i}^{n}\left(a^{\prime}\right) \mid t_{i}\right) .
\end{aligned}
$$

Equations (8) and (9) imply that when $a^{\prime}=b-d+\varepsilon_{n}$ and $t_{i}>\tilde{t}_{i}+\psi$, the left-hand side of (10) is greater than $\frac{\lambda \psi}{2} \gamma_{i}$, while the right-hand side is less than $\frac{1}{4}\left(\frac{\lambda \psi}{2} \gamma_{i}\right)$. Thus, no types $t_{i}>\tilde{t}_{i}+\psi$ use actions less than or equal to $b-d+\varepsilon_{n}$.

We now proceed to show by induction that the same conclusion holds for higher actions on $(b-d, b+d)$ as well. Consider a positive integer $l \geq 2$ such that $b-d+l \varepsilon_{n}<b+d$, and suppose that no types $t_{i}>\tilde{t}_{i}+\psi$ use actions less than or equal to $b-d+(l-1) \varepsilon_{n}$ (which we have just established for $l=2$ ). Consider the change in the left-hand side of (10) when we increase $a^{\prime}$ from $b-d+\varepsilon_{n}$ to $b-d+l \varepsilon_{n}$. First, $E\left[\Delta v_{i}(b+d, \mathbf{t}) \mid t_{i}, W_{i}^{n}(b+d) \backslash W_{i}^{n}\left(a^{\prime}\right)\right]$ increases by A3. Second, $\operatorname{Pr}\left(W_{i}^{n}(b+d) \backslash W_{i}^{n}\left(a^{\prime}\right) \mid t_{i}\right)$ decreases by at most the left-hand side of (6). Thus,

$$
\operatorname{Pr}\left(W_{i}^{n}(b+d) \backslash W_{i}^{n}\left(a^{\prime}\right) \mid t_{i}\right)>\frac{1}{2} \gamma_{i}
$$

when $a^{\prime}=b-d+l \varepsilon_{n}$. Given (9), a lower bound on the left-hand side of (10) is $\frac{1}{2}\left(\frac{\lambda \psi}{2} \gamma_{i}\right)$, still greater than the upper bound of the right-hand side of (10), $\frac{1}{4}\left(\frac{\lambda \psi}{2} \gamma_{i}\right)$. We conclude that for $i \in K$, no types $t_{i}>\tilde{t}_{i}+\psi$ use actions less than or equal to $b-d+l \varepsilon_{n}$. By induction, for all $i \in K$, and all $t_{i} \geq \tilde{t}_{i}+\psi, \beta_{i}^{n}\left(t_{i}\right)>b+d-\varepsilon_{n}$, and Claim 2 is established.

Claim 2 shows that when players $i \in K$ realize types on the upper part of the set $\tilde{S}_{i}$, these types strictly prefer to defeat the mass of opponent types by using action $b+d$. This contradicts the hypothesis of uniform convergence (outside of $E$ ) to strategies that violate (4). Q.E.D. 
LEMMA 6: Consider an auction game satisfying A1-A3, (2)-(3), and the SCC. Let $\boldsymbol{\beta}^{*}(\mathbf{t})$ be the limit of a convergent sequence of nondecreasing PSNE strategies to finite-action games, $\boldsymbol{\beta}^{n}(\mathbf{t})$, that satisfy Lemma 4 (ii). (i) If $a_{i}^{n} \rightarrow b$ and $U_{i}\left(a_{i} t_{i} ; \beta_{-i}^{*}(\cdot)\right)$ is continuous at $a_{i}=b, U_{i}\left(a_{i}^{n}, t_{i} ; \beta_{-i}^{n}(\cdot)\right)$ converges to $U_{i}\left(b, t_{i} ; \beta_{-i}^{*}(\cdot)\right)$. (ii) For all $i$ and almost every $t_{i} U_{i}\left(a_{i} t_{i} ; \beta_{-i}^{*}(\cdot)\right)$ is continuous in $a_{i}$ at $a_{i}=\beta_{i}^{*}\left(t_{i}\right)$.

PROOF: Part (i): First, note that

$$
\begin{aligned}
& U_{i}\left(b, t_{i} ; \beta_{-i}^{*}(\cdot)\right)-U_{i}\left(a_{i}^{n} \beta_{i}^{n}\left(t_{i}\right), t_{i} ; \beta_{-i}^{n}(\cdot)\right) \\
& =\left[U_{i}\left(b, t_{i} ; \beta_{-i}^{*}(\cdot)\right)-U_{i}\left(a_{i}^{n}, t_{i} ; \beta_{-i}^{*}(\cdot)\right)\right]+\left[U_{i}\left(a_{i}^{n}, t_{i} ; \beta_{-i}^{*}(\cdot)\right)-U_{i}\left(a_{i}^{n}, t_{i} ; \beta_{-i}^{n}(\cdot)\right)\right] .
\end{aligned}
$$

The first term of the right-hand side of (12) goes to zero as $n$ gets large by continuity of $U_{i}\left(a_{i}, t_{i} ; \beta_{-i}^{*}(\cdot)\right)$ in $a_{i}$ at $b$. So it remains to consider the second term of the right-hand side of (12). This term converges to zero if $U_{i}\left(a_{i} t_{i} ; \beta_{-i}^{n}(\cdot)\right)$ converges uniformly (across $a_{i}$ in a neighborhood of b) to $U_{i}\left(a_{i} t_{i} ; \beta_{-i}^{*}(\cdot)\right)$. But uniform convergence follows since $f, \underline{v}_{i}$, and $\Delta v_{i}$ are bounded and since $\operatorname{Pr}\left(W_{i}^{*}\left(a_{i}\right)\right)$ is continuous at $a_{i}=b$, and further, $\boldsymbol{\beta}_{-i}^{n}(\cdot)$ converges uniformly to $\beta_{-i}^{*}(\cdot)$ except on a set of arbitrarily small measure (details are in the working paper Athey (1997)).

Part (ii): Since $\bar{v}_{i}$ and $\underline{v}_{i}$ are continuous, whenever $\operatorname{Pr}\left(W_{i}^{*}\left(a_{i}\right)\right)$ is continuous at $a_{i}=b$, player $i$ 's expected payoffs are continuous there as well. Consider $b \in \mathcal{A}_{i}$. Suppose first that $\operatorname{Pr}\left(\tau_{i}^{\tau^{*}}(b)\right)=0$, which implies $\operatorname{Pr}\left(\tau_{i}^{W^{*}}(b)\right)=0$ as well (recall ties are broken randomly). This in turn implies that $\operatorname{Pr}\left(W_{i}^{*}\left(a_{i}\right)\right)$ is continuous at $a_{i}=b$. Suppose second that $\operatorname{Pr}\left(\tau_{i}^{L^{*}}(b)\right)>0$ (this of course implies $b>Q$ ). Then, Lemma 5 establishes that at most a single type of player $i$ uses action $b$. Since $\operatorname{Pr}\left(\tau_{i}^{L^{*}}(b)\right)>0$ for only a countable number of actions $b$, the set of types who face discontinuities has measure zero. $\quad$ Q.E.D.

PROOF OF THEOREM 6: Following the proof of Theorem 2, consider a sequence of games 
with successively finer finite action sets, indexed by $\{n\}$. Restrict attention to sequences of PSNE to these games, $\left\{\beta^{n}(\mathbf{t})\right\}$, that satisfy condition (ii) of Lemma 4 and converge to a set of strategies denoted $\beta^{*}(\mathbf{t})$. Applying the logic of Theorem 2, so long as (i) $U_{i}\left(a_{i}, \beta_{-i}^{*}(\cdot), t_{i}\right)$ is continuous in $a_{i}$ at $a_{i}=\beta_{i}^{*}\left(t_{i}\right)$, and (ii) $U_{i}\left(\beta_{i}^{n}\left(t_{i}\right), t_{i} ; \beta_{-i}^{n}(\cdot)\right)$ converges to $U_{i}\left(\beta_{i}^{*}\left(t_{i}\right), \beta_{-i}^{*}(\cdot), t_{i}\right), \beta_{i}^{*}\left(t_{i}\right)$ is a best response of player $i$, type $t_{i}$ when opponents use $\beta_{-i}^{*}(\cdot)$. But Lemma 6 establishes conditions (i) and (ii) hold for almost every type, and we are done.

PROOF OF THEOREM 7: We make frequent use of the five facts presented at the beginning of Section 3.2. We add three more: (vi) if $f\left(\mathbf{t}_{-i} \mid y\right)$ is log-supermodular and $h(\mathbf{t})$ is nondecreasing, then $\int h(\mathbf{t}) f\left(\mathbf{t}_{-i} \mid y\right) d \mathbf{t}_{-i}$ is nondecreasing in $y$ (Milgrom and Weber (1982)); (vii) $\varphi_{i}(\mathbf{a})$ is log-supermodular for the first-price auction game; (viii) if $\varphi_{i}(\mathbf{a})$ and $f(\mathbf{t})$ are logsupermodular, then the density of $\mathbf{t}_{-i}$ conditional on $a_{i}$ winning is $\log$-supermodular when opponents use nondecreasing strategies, and further $\operatorname{Pr}\left(\right.$ win with $\left.a_{i} t_{i}\right)$ is log-supermodular.

Fact (viii) follows from facts (ii), (iii), and (v). Fact (vii) requires a short proof. Consider a and $\mathbf{a}^{\prime}$, and suppose that $\varphi_{i}(\mathbf{a}) \varphi_{i}\left(\mathbf{a}^{\prime}\right)>0$. This implies $a_{i} \geq a_{j}$ and $a_{i}^{\prime} \geq a_{j}^{\prime}$ for all $i \neq j$; in turn, that implies that the vectors of bids $\mathbf{a} \vee \mathbf{a}^{\prime}$ and $\mathbf{a} \wedge \mathbf{a}^{\prime}$ must each give player $i$ at least some probability of winning. Let $S$ be the set of opponents $j$ that ties for winner with $i$ given a, and likewise let $S^{\prime}$ be the set that ties with $i$ given $\mathbf{a}^{\prime}$. If $a_{i}>a_{i}^{\prime}$, the same set $S$ ties given $\mathbf{a} \vee \mathbf{a}^{\prime}$, and a set smaller than $S^{\prime}$ ties given $\mathbf{a} \wedge \mathbf{a}^{\prime}$, since for some opponents $j$ we may have $a_{j}<a_{j}^{\prime}$. If $a_{i}=a_{i}^{\prime}$, the set $S \cup S^{\prime}$ ties given $\mathbf{a} \vee \mathbf{a}^{\prime}$, while the set $S \cap S^{\prime}$ ties given $\mathbf{a} \wedge \mathbf{a}^{\prime}$. Since $\left|S \cap S^{\prime}\right| S \cup S^{\prime}|\leq| S|| S^{\prime} \mid$, $\varphi_{i}\left(\mathbf{a} \vee \mathbf{a}^{\prime}\right) \varphi_{i}\left(\mathbf{a} \wedge \mathbf{a}^{\prime}\right) \geq \varphi_{i}(\mathbf{a}) \varphi_{i}\left(\mathbf{a}^{\prime}\right)$

Now consider first-price auctions in the affiliated private values setting. Each player's 
expected utility can be written $V_{i}\left(t_{i}-a_{i}\right) \cdot \operatorname{Pr}\left(\right.$ win with $\left.a_{i} \mid t_{i}\right)$. If both terms of this expression are logsupermodular, the objective is log-supermodular and the SCC holds. If $V_{i}(0) \geq 0$ and $\ln \left(V_{i}\right)$ is concave, $V_{i}$ is log-supermodular in $\left(t_{i}, a_{i}\right)$. By facts (vii) and (viii), $\operatorname{Pr}\left(\right.$ win with $\left.a_{i} \mid t_{i}\right)$ is $\log$ supermodular.

The case of positive value interdependence, independent information is similar. Expected utility can be written $E\left[\bar{v}_{i}\left(a_{i} \mathbf{t}\right) \mid\right.$ win with $\left.a_{i}\right] \operatorname{Pr}$ (win with $a_{i}$ ). Since both terms are positive in the relevant region, the probability of winning is nondecreasing in $a_{i}$, and $\bar{v}_{i}\left(a_{i}, \mathbf{t}\right)$ is increasing in $t_{i}$, this expression is supermodular in $\left(a_{i} t_{i}\right)$ if the first term is. By fact (viii), the density of $\mathbf{t}_{-i}$ conditional on $a_{i}$ winning is log-supermodular. Athey (1998b) shows that this, together with $\bar{v}_{i}\left(a_{i}, \mathbf{t}\right)$ is supermodular in $\left(a_{i}, t_{i}\right)$ and in $\left(t_{i}, t_{j}\right)$ for all opponents $j$, implies that the expectation is supermodular. Further, since $\bar{v}_{i}\left(a_{i}, \mathbf{t}\right)$ is nondecreasing in $\mathbf{t}$, fact (vi) implies A3.

Now consider the first-price auction in the general affiliated values model. First, we observe that A3 holds following the logic from the last paragraph. Next, we introduce an additional condition: there exists a function $k_{i}$ and a single random variable, $s_{i}$, such that $U_{i}\left(a_{i}, \boldsymbol{\alpha}_{-i}(\cdot), t_{i}\right)=E_{s_{i}}\left[k_{i}\left(a_{i}, s_{i}, t_{i} ; \boldsymbol{\alpha}_{-i}\right) \mid t_{i}\right]$. If there are only two bidders, this is satisfied when we let $s_{i}=t_{j}$ for $j \neq i$, and let $k_{i}\left(a_{i}, s_{i}, t_{i} ; \alpha_{-}\right)=\bar{v}_{i}\left(a_{i}, t_{i}, s_{j}\right) \cdot \varphi_{i}\left(a_{i}, \alpha_{j}\left(s_{i}\right)\right)$. For more than two bidders, the condition holds if the information structure is conditionally independent common values. In particular, repeated application of Bayes' rule establishes that the requirement is satisfied if we let $s_{i}=Z$ and $k_{i}\left(a_{i}, s_{i}, t_{i} ; \boldsymbol{\alpha}_{-i}\right)=V_{i}\left(s_{i}-a_{i}\right) E\left[\varphi_{i}\left(a_{i}, \boldsymbol{\alpha}_{-i}\left(\mathbf{t}_{-i}\right)\right) \mid s_{i}\right]$. In other contexts, $s_{i}$ might be an order statistic of opponent types, or a sufficient statistic for $\mathbf{t}_{-i}$. The following result can then be used: LEMMA 7: Suppose that for all $i=1, . . I$, there exists a random variable $s_{i}$ and a family of functions $k_{i}\left(\cdot ; \boldsymbol{\alpha}_{-i}\right): \mathbb{R}^{3} \rightarrow \mathbb{R}$, such that (i) $U_{i}\left(a_{i}, \boldsymbol{\alpha}_{-i}(\cdot), t_{i}\right)=E_{s_{i}}\left[k_{i}\left(a_{i}, s_{i}, t_{i} ; \boldsymbol{\alpha}_{-i}\right) \mid t_{i}\right] ;$ (ii) when $\boldsymbol{\alpha}_{-i}(\cdot)$ 
is nondecreasing, $k_{i}\left(a_{i}, s_{i}, t_{i} ; \alpha_{-i}\right)$ is supermodular in $\left(a_{i} ; t_{i}\right)$ and satisfies weak SCP-IR in $\left(a_{i} ; s_{i}\right)$;

(iii) $t_{i}$ and $s_{i}$ are affiliated, and the support of $s_{i}$ does not vary with $t_{i}$. Then the game satisfies $S C C$.

A proof of Lemma 7 can be found in Athey (1998b). Athey (1998b) also shows that in the two-bidder example, $\bar{v}_{i}\left(a_{i}, t_{i}, s_{j}\right) \cdot \varphi_{i}\left(a_{i}, \alpha_{j}\left(s_{i}\right)\right)$ satisfies part (ii) of Lemma 7. Now consider the conditionally independent common values model. Since $V_{i}\left(s_{i}-a_{i}\right) E\left[\varphi_{i}\left(a_{i}, \boldsymbol{\alpha}_{-i}\left(\mathbf{t}_{-i}\right)\right) \mid s_{i}\right]$ does not depend on $t_{i}$, it remains only to show that it satisfies weak SCP-IR in $\left(a_{i} ; s_{i}\right)$. Consider $a_{i}^{H}>a_{i}^{L}$. For $s_{i} \leq a_{i}^{H}$, the returns to using $a_{i}^{H}$ rather than $a_{i}^{L}$ are always negative, since the higher action wins with higher probability, but $V_{i}\left(s_{i}-a_{i}^{H}\right) \leq 0$. So restrict attention to $s_{i}>a_{i}^{H}$; by our normalization assumption, $V_{i}\left(s_{i}-a_{i}\right)$ is positive. Since log-supermodularity is stronger than SCPIR, it suffices to verify log-supermodularity on the restricted domain. So long as $V_{i}$ is $\log$ concave, $V_{i}\left(s_{i}-a_{i}\right)$ is log-supermodular. Further, $E\left[\varphi_{i}\left(a_{i}, \boldsymbol{\alpha}_{-i}\left(\mathbf{t}_{-i}\right)\right) \mid s_{i}\right]=\operatorname{Pr}\left(\right.$ win with $\left.a_{i} \mid s_{i}\right)$ is logsupermodular, since $s_{i}$ is affiliated with all bidder types and $\varphi_{i}$ is $\log$-supermodular.

Now consider multiple-unit auctions. Unfortunately, $\varphi_{i}(\mathbf{a})$ is not $\log$-supermodular in this case. If the types are drawn independently, however, then $\operatorname{Pr}\left(a_{i}\right.$ wins $\left.\mid t_{i}\right)$ does not depend on $t_{i}$, and the objective function becomes $V_{i}\left(t_{i}-a_{i}\right) \cdot \operatorname{Pr}\left(\right.$ win with $\left.a_{i}\right)$, which is $\log$-supermodular if $\ln \left(V_{i}\right)$ is concave.

For the all-pay auction, in the private values case with independent types, expected payoffs are given by $\left[V_{i}\left(t_{i}-a_{i}\right)-V_{i}\left(-a_{i}\right)\right] \operatorname{Pr}\left(a_{i}\right.$ wins $)+V_{i}\left(-a_{i}\right)$. We argue that this objective is supermodular. Since $\operatorname{Pr}\left(a_{i}\right.$ wins $)$ is nonnegative and nondecreasing in $a_{i}$, it is straightforward to verify that the second term of this expression is supermodular if $V_{i}\left(t_{i}-a_{i}\right)-V_{i}\left(-a_{i}\right)$ is nondecreasing in $t_{i}$ (which 
follows since $V_{i}$ is increasing) and supermodular in $\left(a_{i}, t_{i}\right)$. In turn, $V_{i}\left(t_{i}-a_{i}\right)$ is supermodular if and only if it is concave (the bidder is risk averse), as desired. In the positive value interdependence, independent values setting, we established above that $E\left[\bar{v}_{i}\left(a_{i}, \mathbf{t}\right) \mid\right.$ win with $\left.a_{i}\right] \operatorname{Pr}\left(a_{i}\right.$ wins $)$ is supermodular in $\left(a_{i}, t_{i}\right)$; adding the function $V_{i}\left(-a_{i}\right)\left(1-\operatorname{Pr}\left(a_{i}\right.\right.$ wins $\left.)\right)$ does not affect this conclusion, since it does not depend on $t_{i}$.

The analysis of the Bertrand pricing game is similar to the first-price auction. The function $\left(-a_{i}+t_{i}\right) D\left(-a_{i}\right)$ is both supermodular and log-supermodular in $\left(a_{i}, t_{i}\right)$ when demand is downwardsloping. These were the only relevant properties of the utility function for our analysis of firstprice auctions. Similarly, the price dispersion model is analogous to the all-pay auction. 


\section{REFERENCES}

Amann, Erwin and Wolfgang Leininger (1996): “Asymmetric All-Pay Auctions with Incomplete Information: The Two-Player Case," Games and Economic Behavior. 14 (1), 1-18.

Athey, Susan (1997): "Single Crossing Properties and the Existence of Pure Strategy Equilibria in Games of Incomplete Information,” MIT Working Paper Number 97-11.

— (1998a): "Characterizing Properties of Stochastic Objective Functions," MIT Working Paper Number 96-1R.

— (1998b): "Comparative Statics Under Uncertainty: Single Crossing Properties and LogSupermodularity," MIT Working Paper Number 96-22R.

Athey, Susan and Armin Schmutzler (1999): "Innovation and the Evolution of Market Dominance," MIT Working Paper Number 99-18.

Athey, Susan, Kyle Bagwell, and Chris Sanchirico (1998): “Collusion and Price Rigidity," MIT Working Paper Number 98-23.

Bagwell, Kyle and Asher Wolinsky (1997): "Game Theory and Industrial Organization Theory," Mimeo, Columbia University.

Bajari, Patrick (1997): "First-Price Auctions with Asymmetric Bidders: Theory and Applications," Ph.D. Thesis, University of Minnesota.

Baye, M.R., D. Kovenock, and C.G. de Vries (1993): "Rigging the Lobbying Process: An Application of the All-Pay Auction," American Economic Review, 83, 289-294.

Becker, Gary (1983): “A Theory of Competition Among Pressure Groups for Political Influence," Quarterly Journal of Economics 98, 371-400.

Dasgupta, Partha and Eric Maskin (1986): “The Existence of Equilibrium in Discontinuous Economic Games, I: Theory," Review of Economic Studies, 53, 1-26. 
Diamond, Peter (1982): “Aggregate Demand Management in Search Equilibrium,” Journal of Political Economy, 90, 881-894.

Fudenberg, Drew and Jean Tirole (1991): Game Theory. Cambridge: MIT Press.

Fullerton, Richard, and Preston McAfee (1999): “Auctioning Entry Into Tournaments," Journal of Political Economy, 107, 573-605.

Katz, Michael and Carl Shapiro (1986): "Technology Adoption in the Presence of Network Externalities," Journal of Political Economy, 94, 822-841.

Khan, Ali and Yeneng Sun (1995): "Pure strategies in games with private information," Journal of Mathematical Economics, 24, 633-653.

— (1996): "Nonatomic games on Loeb spaces," Proceedings of the National Academy of Sciences, 93, 15518-15521.

- (1997): "On Loeb Measure Spaces and their Significance for Non-Cooperative Game Theory," in M. Alber et al (eds.), Current and Future Directions in Applied Mathematics (Berlin: Birkhauser).

Kolmogorov, A.N. and S.V. Fomin (1970), Introductory Real Analysis, New York: Dover Publications.

Krishna, Vijay and John Morgan (1997): "An Analysis of the War of Attrition and the All-Pay Auction," Journal of Economic Theory, 72, 343-62.

Li, Tong, Isabelle Perrigne, and Quang Vuong, (1999): "Conditionally Independent Private Information in OCS Wildcat Auctions," forthcoming, Journal of Econometrics.

Lizzeri, Alessandro and Nicola Persico (1997): “Uniqueness and Existence of Equilibrium in Auctions with a Reserve Price," Mimeo, UCLA.

Lebrun, Bernard (1997): "First-price Auction in the Asymmetric N Bidder Case," Mimeo, 
Department of Economics, Universite Leval, Sainte-Foy, QC, Canada.

- (1996): "Existence of an Equilibrium in First-price Auctions," Economic Theory, 7, 421-43.

Maggi, Giovanni (1999): "The value of commitment with imperfect observability and private information," RAND Journal of Economics, 30, 555-574.

Marshall, Robert, Michael Meurer, Jean-Francois Richard, and Walter Stromquist (1994): "Numerical Analysis of Asymmetric First-price Auctions," Games and Economic Behavior, 7, 193- 220.

Maskin, Eric and John Riley (1999): "Equilibrium in Sealed High Bid Auctions," Mimeo, Harvard University.

Matthews, S. and L. Mirman (1983): "Equilibrium Limit Pricing: the Effects of Private Information and Stochastic Demand," Econometrica, 51, 981-994.

Milgrom, Paul, and John Roberts (1990): "Rationalizability, Learning, and Equilibrium in Games with Strategic Complementarities," Econometrica, 58, 1255-1277.

Milgrom, Paul, and Chris Shannon (1994): "Monotone Comparative Statics," Econometrica, 62, $157-180$.

Milgrom, Paul, and Robert Weber (1982): "A Theory of Auctions and Competitive Bidding," Econometrica, 50, 1089-1122.

— (1985): "Distributional Strategies for Games with Incomplete Information," Mathematics of Operations Research, 10, 619-632.

O'Neill, B. (1986): "International Escalation and the Dollar Auction," Journal of Conflict Resolution, 30, 33-50.

Pesendorfer, Wolfgang and Jeroen Swinkels (1997): “The Loser's Curse and Information Aggregation in Common Value Auctions," Econometrica, 65, 1247-1282. 
Radner, Roy, and Rosenthal, Robert W. (1982): "Private Information and Pure-Strategy Equilibria," Mathematics of Operations Research, 7, 401-409.

Reny, Philip J. (1999): “On the Existence of Pure and Mixed Strategy Nash Equilibria in Discontinuous Games,” Econometrica 67, 1029-56.

Royden, H.L. (1988): Real Analysis, MacMillan: New York.

Simon, Leo and William Zame (1990): “Discontinuous Games and Endogenous Sharing Rules," Econometrica, 58, 861-72.

Spulber, Daniel (1995): “Bertrand Competition When Rivals' Costs are Unknown,” Journal of Industrial Economics, XLIII, 1-11.

Topkis, Donald (1978): "Minimizing a Submodular Function on a Lattice," Operations Research, 26, 305-321.

-(1979): "Equilibrium Points in Nonzero-Sum n-person Submodular Games," Siam Journal of Control and Optimization, 17, 773-787.

Varian, Hal R. (1980): “A Model of Sales,” American Economic Review, 70, 651-59.

Vives, Xavier (1990): “Nash Equilibrium with Strategic Complementarities," Journal of Mathematical Economics, 19, 305-21.

Weber, Robert (1994): "Equilibrium in Non-Partitioning Strategies," Games and Economic Behavior, 7, 286-294. 


\section{FOOTNOTES}

${ }^{1}$ I am grateful to seminar participants at Arizona State, Columbia, Maryland, MIT, New York University, University of Pennsylvania, Pennsylvania State, Pittsburgh, Princeton, Stanford GSB, UCLA, UC-Berkeley, Toulouse, the 1997 Summer Meetings of the Econometric Society, as well as Kyle Bagwell, Abhijit Banerjee, Prajit Dutta, Glenn Ellison, Ken Judd, Jonathan Levin, Eric Maskin, Preston McAfee, Paul Milgrom, John Roberts, Lones Smith, Jeroen Swinkels, and Bob Wilson for useful conversations, and to Jonathan Dworak, Steven Schulenberg, Luis Ubeda, and Jennifer Wu for excellent research assistance. Nancy Stokey and three anonymous referees provided suggestions which greatly improved the exposition of the paper. I would like to thank the National Science Foundation (Grant No. SBR-9631760) for financial support.

${ }^{2}$ That is, when choosing between a low action and a high action, if a low type of player $i$ weakly (strictly) prefers the higher action, then all higher types of agent $i$ weakly (strictly) prefers the higher action as well.

${ }^{3}$ More precisely, Milgrom and Weber (1985) show that pure strategy equilibria exist when type spaces are atomless and players choose from a finite set of actions, types are independent conditional on some common state variable (which is finite-valued), and each player's utility function depends only on his own type, the other players' actions, and the common state variable (the utility cannot depend on the other players' types directly). They also require a condition which they call "continuity of information."

${ }^{4}$ Khan and Sun (1996) show further that if the type distributions are taken to be atomless on a special class of measure spaces, called hyperfinite Loeb spaces, existence of PSNE can be obtained when actions are drawn from the continuum (again maintaining continuity of payoffs and independence of types).

${ }^{5}$ Dasgupta and Maskin (1986) provide sufficient conditions for existence of mixed strategy equilibria in games with finite types. Simon and Zame (1990) analyze existence of mixed strategy equilibria (and epsilon-equilibria) when tie-breaking rules are endogenous. More recently, Reny (1999) establishes existence of PSNE in general classes of games with discontinuous payoffs, under additional regularity assumptions and a condition called "betterreply security."

${ }^{6}$ For asymmetric independent private values auctions, see Maskin and Riley (1999), Lebrun (1996, 1997), and Bajari (1997); for affiliated private values or common values with independent signals, see Maskin and Riley 
(1999). Pesendorfer and Swinkels (1997) study symmetric, uniform-price common value auctions for multiple units. Lizzeri and Persico (1997) have independently shown that a condition closely related to the single crossing condition is sufficient for existence and uniqueness of equilibrium in two-player auction games with heterogeneous bidders, but their approach only extends to more than two players under symmetry.

${ }^{7}$ Weber (1994) studies mixed strategy equilibria in a class of auction games when types are not affiliated, so that the SCC fails.

${ }^{8}$ In games with finite actions, Assumption A1 can be relaxed to allow for mass points at the lower end of the distribution, so long as for each player, there exists a $k>t_{-j}$ such that the lowest action chosen by player $j$ is chosen throughout the region $\left[t_{j}, k\right)$.

${ }^{9}$ Athey (1997) shows that Theorem 2 can be easily extended to show that if every finite-action game has an equilibrium in strategies of bounded variation, the continuum-action game will as well.

${ }^{10}$ Khan and Sun $(1996,1997)$ show that the use of atomless Loeb measure spaces for the types, as an alternative to Lebesgue, can restore the applicability of limiting arguments.

11 The operations "meet" $(\vee)$ and “join" $(\wedge)$ are defined for product sets as follows: $\mathbf{x} \vee \mathbf{y}=\left(\max \left(x_{1}, y_{1}\right), \ldots, \max \left(x_{n}, y_{n}\right)\right)$ and $\mathbf{x} \wedge \mathbf{y}=\left(\min \left(x_{1}, y_{1}\right), \ldots, \min \left(x_{n}, y_{n}\right)\right)$

${ }^{12}$ For the case where payoffs are supermodular, the assumption that types are affiliated can be weakened; what is actually required is that for each $i, \int_{S} f\left(\mathbf{t}_{-i} \mid t_{i}\right) d \mathbf{t}_{-i}$ is nondecreasing in $t_{i}$ for all sets $S$ whose indicator function is nondecreasing in $\mathbf{t}_{-i}$. See Athey (1998a).

${ }^{13}$ See Fudenberg and Tirole $(1991,215-216)$ for an example with linear demand and incomplete information about cost.

${ }^{14}$ The interpretation of the latter condition is that the elasticity of demand is a non-increasing function of the other firms' prices. As discussed in Milgrom and Roberts (1990b), demand functions which satisfy this criteria include logit, CES, transcendental logarithmic, and a set of linear demand functions (see Topkis (1979)).

${ }^{15}$ This result follows as a corollary of Lemma 7 in the Appendix.

${ }^{16}$ Intermediate outcomes could also be considered; the arguments used to establish existence extend naturally. 
${ }^{17}$ When a player's payoffs satisfy the single crossing property, only direct mechanisms in which the allocation rule is monotonic can be incentive compatible; then, we can let the player's announcement of type be her action, and redefine payoffs to incorporate the allocation rule of the mechanism.

${ }^{18}$ While quasi-Newton methods might at first seem computationally burdensome, there are potentially large computational benefits to using an analytic Jacobian. In particular, the point at which player $i$ jumps to action $A_{m}$, denoted $x_{m}^{i}$, affects only the following elements of the best response of opponent $j \neq i: x_{m-1}^{j}, x_{m}^{j}$, and $x_{m+1}^{j}$. Thus, the Jacobian (of dimension $M \cdot I \times M \cdot I$ ) can be computed with only $I \cdot 3$ function calls.

${ }^{19}$ Our analysis can be extended to multiple-unit demands, so long as bidder preferences are summarized in a one-dimensional type, but this requires an extension to allow for more than one discontinuity in the payoffs, as the bidder goes from winning $n$ objects to $n+1$. 


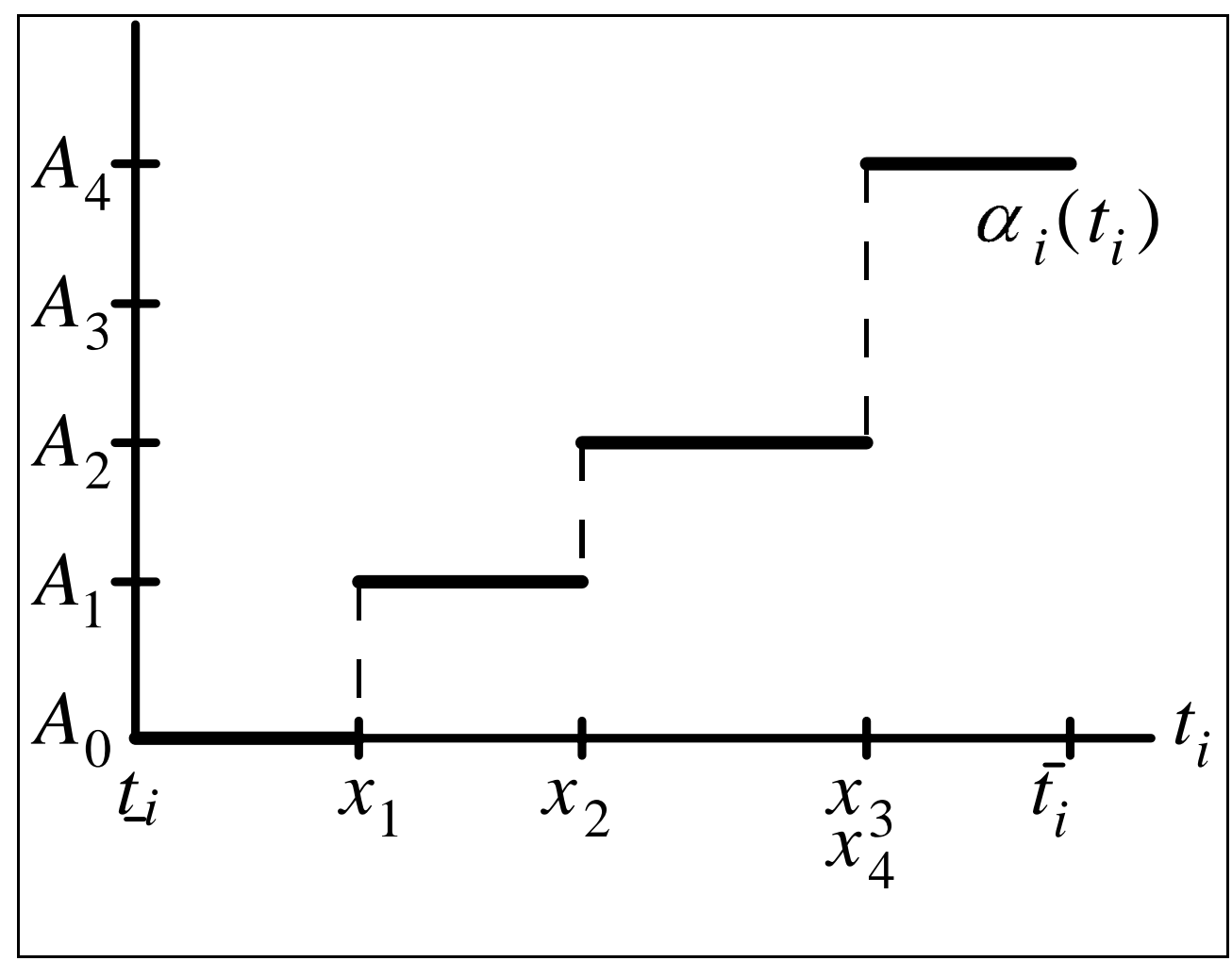




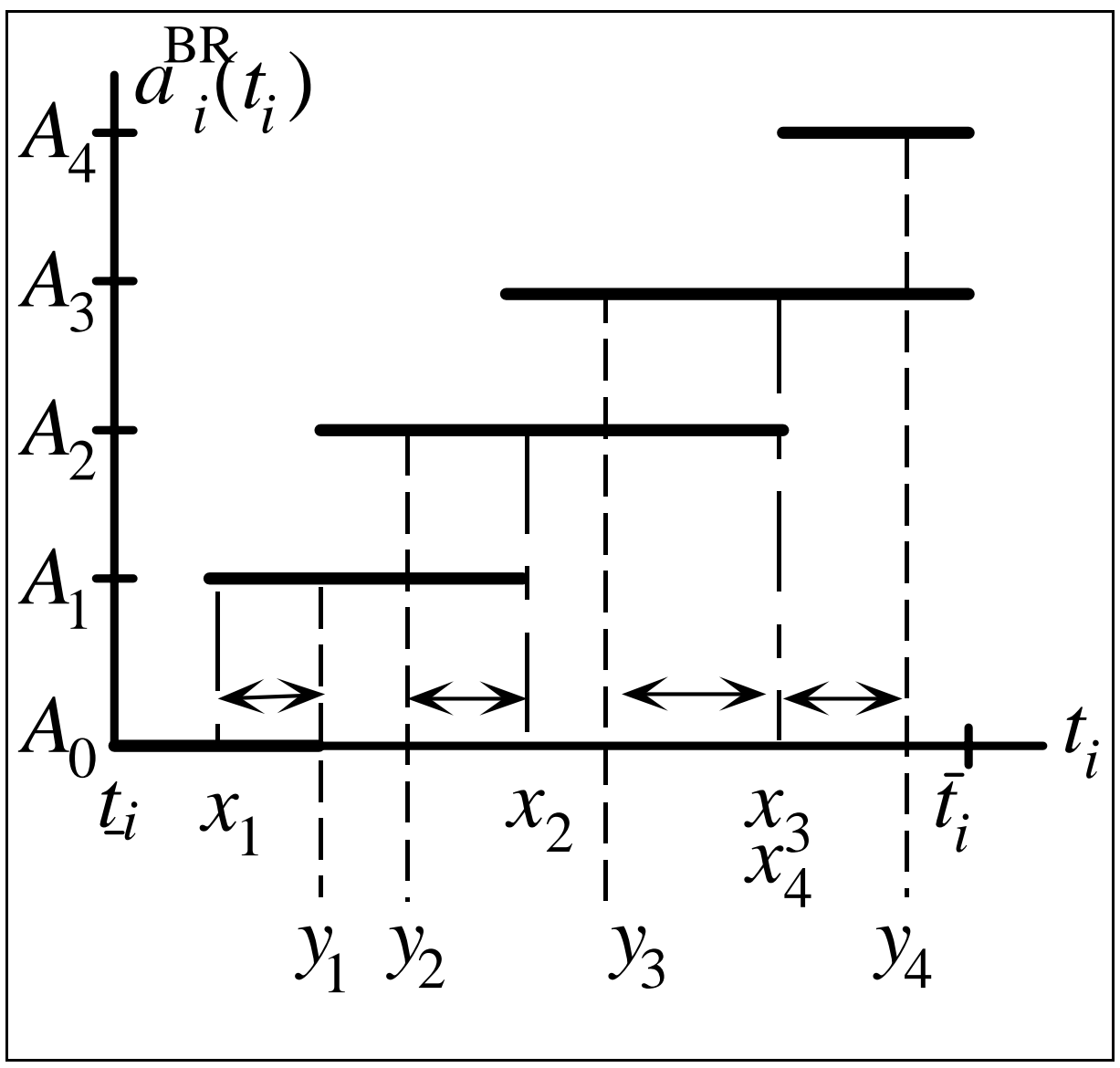




\section{CAPTIONS}

FIGURE 1: The elements of the vector $\mathbf{x}$ specify when the agent "jumps" to a higher action.

FIGURE 2: The set $a_{i}^{B R}\left(t_{i}\right)$ is nondecreasing in the Strong Set Order. The vectors $\mathbf{x}$ and $\mathbf{y}$ represent "jump points" corresponding to optimal strategies. The arrows indicate convex combinations of $\mathbf{x}$ and $\mathbf{y}$. 